\title{
Gene regulatory network inference by point-based Gaussian approximation filters incorporating the prior information
}

Bin Jia ${ }^{1}$ and Xiaodong Wang ${ }^{2^{*}}$

\begin{abstract}
The extended Kalman filter (EKF) has been applied to inferring gene regulatory networks. However, it is well known that the EKF becomes less accurate when the system exhibits high nonlinearity. In addition, certain prior information about the gene regulatory network exists in practice, and no systematic approach has been developed to incorporate such prior information into the Kalman-type filter for inferring the structure of the gene regulatory network. In this paper, an inference framework based on point-based Gaussian approximation filters that can exploit the prior information is developed to solve the gene regulatory network inference problem. Different point-based Gaussian approximation filters, including the unscented Kalman filter (UKF), the third-degree cubature Kalman filter ( $\left.\mathrm{CKF}_{3}\right)$, and the fifth-degree cubature Kalman filter $\left(\mathrm{CKF}_{5}\right)$ are employed. Several types of network prior information, including the existing network structure information, sparsity assumption, and the range constraint of parameters, are considered, and the corresponding filters incorporating the prior information are developed. Experiments on a synthetic network of eight genes and the yeast protein synthesis network of five genes are carried out to demonstrate the performance of the proposed framework. The results show that the proposed methods provide more accurate inference results than existing methods, such as the EKF and the traditional UKF.
\end{abstract}

Keywords: Gene regulatory network; Point-based Gaussian approximation filters; Network prior information; Sparsity; Iterative thresholding

\section{Introduction}

Inferring gene regulatory network (GRN) has become one of the most important missions in system biology. Genome-wide expression data is widely used due to the development of several high-throughput experimental technologies. The gene regulatory network can be inferred from a number of gene expression samples taken over a period of time. Modeling of GRN is required before its structure can be inferred. Common dynamical modeling methods of GRN include Bayesian networks [1], Boolean networks [2], ordinary differential equations [3], state-space models $[4,5]$, and so on. Various approaches based on different models have been used to infer the network from observed gene expression data, such as the

\footnotetext{
*Correspondence: wangx@ee.columbia.edu

2 Department of Electrical Engineering, Columbia University, New York, NY 10027, USA

Full list of author information is available at the end of the article
}

Markov Chain Monte Carlo (MCMC) methods for the dynamic Bayesian network model [6] and the ordinary differential equation model [7], as well as the Kalman filtering methods for the state-space model $[4,8]$ and the ordinary differential equation model [3]. Some survey papers can be found in [9-12].

Due to the 'stochastic' nature of the gene expression, the Kalman filtering approach based on the state-space model is one of the most competitive methods for inferring the GRN. The Kalman filter is optimal for linear Gaussian systems. However, the GRN is generally highly nonlinear. Hence, advanced filtering methods for nonlinear dynamic systems should be considered. The extended Kalman filter (EKF) is probably the most widely used nonlinear filter which uses the first-order Taylor series expansion to linearize the nonlinear model. However, the accuracy of the EKF is low when the system is highly nonlinear or contains large uncertainty. The point-based

\section{是 Springer}

(c) 2013 Jia and Wang; licensee Springer. This is an Open Access article distributed under the terms of the Creative Commons Attribution License (http://creativecommons.org/licenses/by/2.0), which permits unrestricted use, distribution, and reproduction in any medium, provided the original work is properly cited. 
Gaussian approximation filters have been recently proposed to improve the performance of the EKF, which employ various quadrature rules to compute the integrals involved in the exact Bayesian estimation. Many filters fall into this category, such as the unscented Kalman filter (UKF) [13], the Gauss-Hermite quadrature filter [14], the cubature Kalman filter (CKF) [15], and the sparse-grid quadrature filter [16]. Besides the point-based Gaussian approximation filters, the particle filter has drawn much attention recently [17]. The particle filter uses random particles with weights to represent the probability density function (pdf) in the Bayesian estimation and provides better estimation result than the EKF. The main problem of the particle filter is that the computational complexity is high, and therefore, it is hard to use for high-dimensional problems, such as the problem considered in this paper.

The EKF and the particle filter have been used for the inference of GRN $[4,8,18]$. In this paper, we consider the point-based Gaussian approximation filters. Our main objective is to provide a framework of incorporating network prior information into the filters. For example, some gene regulations may be known [19] from literature and the inference accuracy of GRN can be improved by incorporating the known regulations of the GRN [20]. Integration of the prior knowledge or constraints with the GRN inference algorithm has been introduced to improve the inference result. The DNA motif sequence in gene promoter regions is incorporated in [21] while modeling of transcription factor interactions is incorporated in [22]. As mentioned in [20], experimentally determined physical interactions can be obtained. In addition, the sparsity constraint is frequently used in the inference of the GRN. To the best of the authors' knowledge, the most related work in incorporating the prior information in Bayesian filters is [8]. In that work, rather than directly getting the inference results from the filter, an optimization method is used. In particular, a cost function is used in which the sparsity constraint is enforced. However, the cost function in [8] does not consider the uncertainty of the state in the filtering. That cost function in fact is not coupled well with the filtering algorithm. In addition, it did not consider other kinds of prior information. In this paper, we propose a new framework that incorporates the prior information effectively in the filtering algorithm by solving a constrained optimization problem. Efficient recursive algorithms are provided to solve the associated optimization problem.

The remainder of this paper is organized as follows. In Section 2, the modeling of gene regulatory network is introduced. The point-based Gaussian approximation filters are briefly introduced in Section 3. The proposed new filtering framework is described in Section 4. In Section 5, experimental results are provided. Finally, concluding remarks are given in Section 6.

\section{State-space modeling of gene regulatory network}

The GRN can be described by a graph in which genes are viewed as nodes and edges depict causal relations between genes. The structure of GRN reveals the mechanisms of biological cells. Analyzing the structure of GRN will pave the way for curing various diseases [23]. The learning of GRN has drawn much attention recently due to the availability the microarray data. By analyzing collected gene expression levels over a period of time, one can identify various regulatory relations between different genes. To facilitate the analysis of the GRN, modeling of GRN is required. Different models can be used, such as Bayesian networks [1], Boolean networks [2], ordinary differential equation [3], and state-space model [4,5]. The state-space model has been widely used because it incorporates noise and can make use of computationally efficient filtering algorithms [5]. Thus, we also use the state-space modeling of GRN in this paper.

Under the discrete-time state-space modeling of the gene regulatory networks, the network evolution from time $k$ to time $k-1$ can be described by

$$
\boldsymbol{x}_{k}=\boldsymbol{f}\left(\boldsymbol{x}_{k-1}\right)+\boldsymbol{v}_{k},
$$

where $\boldsymbol{x}_{k}=\left[x_{1, k}, \ldots, x_{n, k}\right]^{T}$ is the state vector and $x_{i, k}$ denotes the gene expression level of the $i$-th gene at time $k$. $f$ is a nonlinear function that characterizes the regulatory relationship among the genes. $v_{k}$ is the state noise and it is assumed to follow a Gaussian distribution with mean $\mathbf{0}$ and covariance matrix $\boldsymbol{Q}_{k}$, i.e., $\boldsymbol{v}_{k} \sim \mathcal{N}\left(\mathbf{0}, \boldsymbol{Q}_{k}\right)$.

Following [8], we use the following nonlinear function in the state Equation (1):

$$
\boldsymbol{f}(\boldsymbol{x})=\boldsymbol{A} \boldsymbol{g}(\boldsymbol{x}),
$$

with

$$
\boldsymbol{g}(\boldsymbol{x})=\left[\begin{array}{c}
g_{1}\left(x_{1}\right) \\
\vdots \\
g_{n}\left(x_{n}\right)
\end{array}\right]
$$

and

$$
g_{i}(x)=\frac{1}{1+e^{-\mu_{i} x}} .
$$

In (2), $\boldsymbol{A}$ is the regulatory coefficient matrix with $a_{i j}$ denoting the regulation coefficient from gene $j$ to gene $i$. Note that a positive coefficient $a_{i j}$ indicates that gene $j$ activates gene $i$ and a negative $a_{i j}$ indicates that gene $j$ represses gene $i$. In (4), $\mu_{i}$ is a parameter. Note that $\boldsymbol{A}$ and $\mu_{i}$ are unknown parameters. The discrete-time nonlinear stochastic dynamic system [24] shown in Eqs. (1)-(3) have been successfully used to describe the GRN $[4,8]$. Equation (4) is also called Sigmoid function which is frequently used since it is consistent with the fact that all concentrations get saturated at some point in time [25]. 
The Sigmoid function has been used in modeling GRN to verify various methods, such as artificial neural network [26], simulated annealing and clustering algorithm [27], extended Kalman filter [4], particle filter [8], and Genetic programming and Kalman filtering [25].

For the measurement model, we consider the following general nonlinear observation equation

$$
\boldsymbol{y}_{k}=\boldsymbol{h}\left(\boldsymbol{x}_{k}\right)+\boldsymbol{n}_{k}
$$

where $\boldsymbol{h}(\cdot)$ is some nonlinear function, $\boldsymbol{n}_{k}$ is the measurement noise, which is assumed to follow the Gaussian distribution with mean $\mathbf{0}$ and covariance matrix $\boldsymbol{R}_{k}$, i.e., $\boldsymbol{n}_{k} \sim \mathcal{N}\left(\mathbf{0}, \boldsymbol{R}_{k}\right)$. For example, if the noise corrupted expression levels are observed, then $\boldsymbol{h}(\boldsymbol{x})=\boldsymbol{x}$.

\section{Network inference using point-based Gaussian approximation filters}

\subsection{Gaussian approximation filters}

In this section, the framework of point-based Gaussian approximation filters for the state-space dynamic model is briefly reviewed. We consider the state-space model consisting of the state Equation (1) and the measurement Equation (5). We denote $\boldsymbol{y}^{k} \triangleq\left[\boldsymbol{y}_{1}, \ldots, \boldsymbol{y}_{k}\right]$.

The optimal Bayesian filter is composed of two steps: prediction and filtering. Specifically, given the prior pdf $p\left(\boldsymbol{x}_{k-1} \mid \boldsymbol{y}^{k-1}\right)$ at time $k-1$, the predicted conditional pdf $p\left(\boldsymbol{x}_{k} \mid \boldsymbol{y}^{k-1}\right)$ is given by

$$
p\left(\boldsymbol{x}_{k} \mid \boldsymbol{y}^{k-1}\right)=\int p\left(\boldsymbol{x}_{k} \mid \boldsymbol{x}_{k-1}\right) p\left(\boldsymbol{x}_{k-1} \mid \boldsymbol{y}^{k-1}\right) \mathrm{d} \boldsymbol{x}_{k-1} .
$$

After the measurement at time $k$ becomes available, the filtered pdf is given by

$$
p\left(\boldsymbol{x}_{k} \mid \boldsymbol{y}^{k}\right)=\frac{p\left(\boldsymbol{y}_{k} \mid \boldsymbol{x}_{k}\right) p\left(\boldsymbol{x}_{k} \mid \boldsymbol{y}^{k-1}\right)}{\int p\left(\boldsymbol{y}_{k} \mid \boldsymbol{x}_{k}\right) p\left(\boldsymbol{x}_{k} \mid \boldsymbol{y}^{k-1}\right) \mathrm{d} \boldsymbol{x}_{k}} .
$$

The pdf recursions in (6) and (7) are in general computationally intractable unless the system is linear and Gaussian. The Gaussian approximation filters approximate (6) and (7) by invoking Gaussian assumptions. Specifically, the first assumption is that given $y^{k-1}$, $\boldsymbol{x}_{k-1}$ has a Gaussian distribution, i.e., $\boldsymbol{x}_{k-1} \mid \boldsymbol{y}^{k-1} \sim$ $\mathcal{N}\left(\hat{\boldsymbol{x}}_{k-1 \mid k-1}, \boldsymbol{P}_{k-1 \mid k-1}\right)$. The second assumption is that $\left(\boldsymbol{x}_{k}, \boldsymbol{y}_{k}\right)$ are jointly Gaussian given $\boldsymbol{y}^{k-1}$.

It then follows from the second assumption that given $\boldsymbol{y}^{k-1}, \boldsymbol{x}_{k}$ has a Gaussian distribution, i.e., $\boldsymbol{x}_{k} \mid \boldsymbol{y}^{k-1} \sim$ $\mathcal{N}\left(\hat{\boldsymbol{x}}_{k \mid k-1}, \boldsymbol{P}_{k \mid k-1}\right)$. Using (1) and the first assumption, we have the predicted mean $\hat{\boldsymbol{x}}_{k \mid k-1}$ and covariance $\boldsymbol{P}_{k \mid k-1}$ given respectively by

$$
\begin{aligned}
\hat{\boldsymbol{x}}_{k \mid k-1} & \triangleq \mathbb{E}\left\{\boldsymbol{x}_{k} \mid \boldsymbol{y}^{k-1}\right\}=\mathbb{E}_{\boldsymbol{x}_{k-1} \mid \boldsymbol{y}^{k-1}}\left\{\boldsymbol{f}\left(\boldsymbol{x}_{k-1}\right)\right\} \\
& =\int \boldsymbol{f}(\boldsymbol{x}) \phi\left(\boldsymbol{x} ; \hat{\boldsymbol{x}}_{k-1 \mid k-1}, \boldsymbol{P}_{k-1 \mid k-1}\right) \mathrm{d} \boldsymbol{x},
\end{aligned}
$$

and

$$
\begin{aligned}
\boldsymbol{P}_{k \mid k-1} \triangleq & \operatorname{Cov}\left\{\boldsymbol{x}_{k} \mid \boldsymbol{y}^{k-1}\right\} \\
= & \mathbb{E}_{\boldsymbol{x}_{k-1} \mid \boldsymbol{y}^{k-1}}\left\{\left(\boldsymbol{f}\left(\boldsymbol{x}_{k-1}\right)-\hat{\boldsymbol{x}}_{k \mid k-1}\right)\right. \\
& \left.\times\left(\boldsymbol{f}\left(\boldsymbol{x}_{k-1}\right)-\hat{\boldsymbol{x}}_{k \mid k-1}\right)^{T}\right\}+\boldsymbol{Q}_{k-1} \\
= & \int\left(\boldsymbol{f}(\boldsymbol{x})-\hat{\boldsymbol{x}}_{k \mid k-1}\right) \\
& \times\left(\boldsymbol{f}(\boldsymbol{x})-\hat{\boldsymbol{x}}_{k \mid k-1}\right)^{T} \boldsymbol{\phi}\left(\boldsymbol{x} ; \hat{\boldsymbol{x}}_{k-1 \mid k-1},\right. \\
& \left.\boldsymbol{P}_{k-1 \mid k-1}\right) \mathrm{d} \boldsymbol{x}+\boldsymbol{Q}_{k-1},
\end{aligned}
$$

where $\phi(x ; \hat{\boldsymbol{x}}, \boldsymbol{P})$ denotes the multivariate Gaussian pdf with mean $\hat{\boldsymbol{x}}$ and covariance $\boldsymbol{P}$.

Then, following the second assumption, given $y^{k}=$ $\left[\boldsymbol{y}^{k-1}, \boldsymbol{y}_{k}\right], \boldsymbol{x}_{k}$ is Gaussian distributed, i.e., $\boldsymbol{x}_{k} \mid \boldsymbol{y}^{k} \sim$ $\mathcal{N}\left(\hat{\boldsymbol{x}}_{k \mid k}, \boldsymbol{P}_{k \mid k}\right)$. Using the conditional property of the multivariate Gaussian distribution, the filtered mean $\hat{\boldsymbol{x}}_{k \mid k}$ and covariance $\boldsymbol{P}_{k \mid k}$ are given respectively by

$$
\begin{aligned}
\hat{\boldsymbol{x}}_{k \mid k} & \triangleq \mathbb{E}\left\{\boldsymbol{x}_{k} \mid \boldsymbol{y}_{k}, \boldsymbol{y}^{k-1}\right\} \\
& =\hat{\boldsymbol{x}}_{k \mid k-1}+\boldsymbol{L}_{k}\left(\boldsymbol{y}_{k}-\hat{\boldsymbol{y}}_{k \mid k-1}\right) \\
\text { and } \boldsymbol{P}_{k \mid k} & \triangleq \operatorname{Cov}\left\{\boldsymbol{x}_{k} \mid \boldsymbol{y}_{k}, \boldsymbol{y}^{k-1}\right\} \\
& =\boldsymbol{P}_{k \mid k-1}-\boldsymbol{L}_{k} \boldsymbol{P}_{k}^{x y},
\end{aligned}
$$

with

$$
\begin{aligned}
\hat{\boldsymbol{y}}_{k \mid k-1}= & \mathbb{E}_{\boldsymbol{x}_{k} \mid \boldsymbol{y}^{k-1}}\left\{\boldsymbol{h}\left(\boldsymbol{x}_{k}\right)\right\} \\
= & \int \boldsymbol{h}(\boldsymbol{x}) \phi\left(\boldsymbol{x} ; \hat{\boldsymbol{x}}_{k \mid k-1}, \boldsymbol{P}_{k \mid k-1}\right) \mathrm{d} \boldsymbol{x}, \\
\boldsymbol{L}_{k}= & \boldsymbol{P}_{k}^{x y}\left(\boldsymbol{R}_{k}+\boldsymbol{P}_{k}^{y y}\right)^{-1}, \\
\boldsymbol{P}_{k}^{x y}= & \mathbb{E}_{\boldsymbol{x}_{k} \mid \boldsymbol{y}^{k-1}}\left\{\left(\boldsymbol{x}-\hat{\boldsymbol{x}}_{k \mid k-1}\right)\left(\boldsymbol{h}(\boldsymbol{x})-\hat{\boldsymbol{y}}_{k \mid k-1}\right)^{T}\right\} \\
= & \int\left(\boldsymbol{x}-\hat{\boldsymbol{x}}_{k \mid k-1}\right)\left(\boldsymbol{h}(\boldsymbol{x})-\hat{\boldsymbol{y}}_{k \mid k-1}\right)^{T} \\
\boldsymbol{P}_{k}^{y y}= & \left.\left.\mathbb{E}_{\boldsymbol{x}_{k} \mid \boldsymbol{y}^{k-1}\left\{\left(\boldsymbol{h} ; \hat{\boldsymbol{x}}_{k \mid k-1}, \boldsymbol{P}_{k \mid k-1}\right) \mathrm{d} \boldsymbol{x},\right.}, \hat{\boldsymbol{y}}_{k \mid k-1}\right)\left(\boldsymbol{h}(\boldsymbol{x})-\hat{\boldsymbol{y}}_{k \mid k-1}\right)^{T}\right\} \\
= & \int\left(\boldsymbol{h}(\boldsymbol{x})-\hat{\boldsymbol{y}}_{k \mid k-1}\right)\left(\boldsymbol{h}(\boldsymbol{x})-\hat{\boldsymbol{y}}_{k \mid k-1}\right)^{T} \\
& \phi\left(\boldsymbol{x} ; \hat{\boldsymbol{x}}_{k \mid k-1}, \boldsymbol{P}_{k \mid k-1}\right) \mathrm{d} \boldsymbol{x} .
\end{aligned}
$$

\subsection{Point-based Gaussian approximation filters}

The integrals in (8), (9), (12), (14) and (15) are Gaussian type that can be efficiently approximated by various quadrature methods. Specifically, if a set of weighted points $\left\{\left(\gamma_{i}, w_{i}\right), i=1, \ldots, N\right\}$ can be used to approximate the integral

$$
\int \boldsymbol{h}(\boldsymbol{x}) \phi(\boldsymbol{x} ; \mathbf{0}, \boldsymbol{I}) \mathrm{d} \boldsymbol{x} \approx \sum_{i=1}^{N} w_{i} \boldsymbol{h}\left(\boldsymbol{\gamma}_{i}\right)
$$


then the general Gaussian-type integral can be approximated by

$$
\int \boldsymbol{h}(\boldsymbol{x}) \phi(\boldsymbol{x} ; \hat{\boldsymbol{x}}, \boldsymbol{P}) \mathrm{d} \boldsymbol{x} \approx \sum_{i=1}^{N} w_{i} \boldsymbol{h}\left(\boldsymbol{S} \boldsymbol{\gamma}_{i}+\hat{\boldsymbol{x}}\right),
$$

where $\boldsymbol{P}=\boldsymbol{S} \boldsymbol{S}^{T}$ and $\boldsymbol{S}$ can be obtained by Cholesky decomposition or singular value decomposition (SVD).

Using (17), we can then approximate (8) and (9) as follows:

$$
\hat{\boldsymbol{x}}_{k \mid k-1} \approx \sum_{i=1}^{N} w_{i} \boldsymbol{f}\left(\boldsymbol{\xi}_{k-1, i}\right)
$$

and

$$
\begin{aligned}
\boldsymbol{P}_{k \mid k-1} \approx & \sum_{i=1}^{N} w_{i} \boldsymbol{f}\left(\boldsymbol{\xi}_{k-1, i}-\hat{\boldsymbol{x}}_{k \mid k-1}\right) \\
& \times\left(\boldsymbol{\xi}_{k-1, i}-\hat{\boldsymbol{x}}_{k \mid k-1}\right)^{T}+\boldsymbol{Q}_{k-1},
\end{aligned}
$$

where $\xi_{k-1, i}$ is the transformed quadrature point obtained from the covariance decomposition, i.e.,

$$
\begin{aligned}
\boldsymbol{P}_{k-1 \mid k-1} & =\boldsymbol{S}_{k-1} \boldsymbol{S}_{k-1}^{T}, \\
\boldsymbol{\xi}_{k-1, i} & =\boldsymbol{S}_{k-1} \boldsymbol{\gamma}_{i}+\hat{\boldsymbol{x}}_{k-1 \mid k-1} .
\end{aligned}
$$

Similarly, we can approximate (12), (14) and (15) as follows:

$$
\begin{aligned}
\hat{\boldsymbol{y}}_{k \mid k-1} & =\sum_{i=1}^{N} w_{i} \boldsymbol{h}\left(\tilde{\boldsymbol{\xi}}_{k, i}\right), \\
\boldsymbol{P}_{k}^{x y} & =\sum_{i=1}^{N} w_{i}\left(\tilde{\boldsymbol{\xi}}_{k, i}-\hat{\boldsymbol{x}}_{k \mid k-1}\right)\left(\boldsymbol{h}\left(\tilde{\boldsymbol{\xi}}_{k, i}\right)-\hat{\boldsymbol{y}}_{k \mid k-1}\right)^{T}, \\
\boldsymbol{P}_{k}^{y y} & =\sum_{i=1}^{N} w_{i}\left(\boldsymbol{h}\left(\tilde{\boldsymbol{\xi}}_{k, i}\right)-\hat{\boldsymbol{y}}_{k \mid k-1}\right)\left(\boldsymbol{h}\left(\tilde{\boldsymbol{\xi}}_{k, i}\right)-\hat{\boldsymbol{y}}_{k \mid k-1}\right)^{T},
\end{aligned}
$$

where $\tilde{\xi}_{k, i}$ is the transformed point obtained from the decomposition of the predicted covariance, i.e.,

$$
\begin{aligned}
\boldsymbol{P}_{k \mid k-1} & =\tilde{\boldsymbol{S}}_{k} \tilde{\boldsymbol{S}}_{k}^{T}, \\
\tilde{\boldsymbol{\xi}}_{k, i} & =\tilde{\boldsymbol{S}}_{k} \boldsymbol{\gamma}_{i}+\hat{\boldsymbol{x}}_{k \mid k-1} .
\end{aligned}
$$

Various numerical rules can be used to form the approximation in (16), which lead to different Gaussian approximation filters. In particular, the unscented transformation, the Gauss-Hermite quadrature rule, and the sparse-grid quadrature rules are used in the unscented Kalman filter (UKF), the Gauss-Hermite quadrature Kalman filter (GHQF), and the sparse-grid quadrature filter (SGQF), respectively.

Recently, the fifth-degree quadrature filter has been proposed and shown to be more accurate than the thirddegree quadrature filters, such as the UKF and the thirddegree cubature Kalman filter $\left(\mathrm{CKF}_{3}\right)$, when the system is highly nonlinear or contains large uncertainty [16]. In this paper, we consider the UKF, $\mathrm{CKF}_{3}$, and the fifth-degree cubature Kalman filter $\left(\mathrm{CKF}_{5}\right)$. Other filters such as the central difference filter [14] and divided difference filter [28] can also be used. The $\mathrm{CKF}_{5}$ is based on Mysovskikh's method which uses fewer point than the fifth-degree quadrature filter in [16]. In the following, different numerical rules used in (16) are briefly summarized.

\subsubsection{Unscented transform}

In the unscented Kalman filter (UKF), we have $N=2 n+1$ where $n$ is the dimension of $\boldsymbol{x}$. The quadrature points and the corresponding weights are given respectively by

$$
\boldsymbol{\gamma}_{i}=\left\{\begin{array}{lr}
\mathbf{0}, & i=1, \\
\sqrt{(n+\kappa)} \boldsymbol{e}_{i-1}, & i=2, \cdots, n+1, \\
-\sqrt{(n+\kappa)} \boldsymbol{e}_{i-n-1}, & i=n+2, \cdots, 2 n+1,
\end{array}\right.
$$

and

$$
w_{i}=\left\{\begin{array}{lr}
\frac{\kappa}{n+\kappa}, & i=1, \\
\frac{1}{2(n+\kappa)}, & i=2, \cdots, 2 n+1,
\end{array}\right.
$$

where $\kappa$ is a tunable parameter, and $\boldsymbol{e}_{i}$ is the $i$-th $n$ dimensional unit vector in which the $i$-th element is 1 and other elements are 0 .

\subsubsection{Cubature rules}

The left-hand side of (16) can be rewritten as

$$
\int \boldsymbol{h}(\boldsymbol{x}) \phi(\boldsymbol{x} ; \mathbf{0}, \boldsymbol{I}) \mathrm{d} \boldsymbol{x}=\frac{1}{\pi^{n / 2}} \int \boldsymbol{h}(\sqrt{2} \boldsymbol{x}) \exp \left(-\boldsymbol{x}^{T} \boldsymbol{x}\right) \mathrm{d} \boldsymbol{x} .
$$

Consider the integral

$$
I(\boldsymbol{h})=\int \boldsymbol{h}(\boldsymbol{x}) \exp \left(-\boldsymbol{x}^{T} \boldsymbol{x}\right) \mathrm{d} \boldsymbol{x} .
$$

By letting $\boldsymbol{x}=r \boldsymbol{s}$ with $\boldsymbol{s}^{T} \boldsymbol{s}=1$ and $r=\sqrt{\boldsymbol{x}^{T} \boldsymbol{x}}, I(\boldsymbol{h})$ can be rewritten in the spherical-radial coordinate system as

$$
I(\boldsymbol{h})=\int_{0}^{\infty} \int_{U_{n}} \boldsymbol{h}(r \boldsymbol{s}) r^{n-1} \exp \left(-r^{2}\right) \mathrm{d} \sigma(\boldsymbol{s}) \mathrm{d} r,
$$

where $U_{n}=\left\{\boldsymbol{s} \in \boldsymbol{R}^{n}:\|\boldsymbol{s}\|=1\right\}$, and $\sigma(\cdot)$ is the spherical surface measure or the area element on $U_{n}$.

Note that (31) contains two types of integrals: the radial integral $\int_{0}^{\infty} h_{r}(r) r^{n-1} \exp \left(-r^{2}\right) \mathrm{d} r$ and the spherical inte$\operatorname{gral} \int_{U_{n}} \boldsymbol{h}_{s}(\boldsymbol{s}) \mathrm{d} \sigma(\boldsymbol{s})$.

If the radial rule can be approximated by

$$
\int_{0}^{\infty} h_{r}(r) r^{n-1} \exp \left(-r^{2}\right) \mathrm{d} r \approx \sum_{i=1}^{N_{r}} w_{r, i} h_{r}\left(r_{i}\right),
$$

and the spherical integral can be approximated by

$$
\int_{U_{n}} \boldsymbol{h}_{s}(\boldsymbol{s}) \mathrm{d} \sigma(\boldsymbol{s}) \approx \sum_{j=1}^{N_{s}} w_{s, j} \boldsymbol{h}_{s}\left(\boldsymbol{s}_{j}\right)
$$


then (31) can be approximated by

$$
I(\boldsymbol{h}) \approx \sum_{i=1}^{N_{r}} \sum_{j=1}^{N_{s}} w_{r, i} w_{s, j} \boldsymbol{h}\left(r_{i} \boldsymbol{s}_{j}\right) .
$$

A third-degree cubature rule to approximate (29) is obtained by using the third-degree spherical rule and radial rule [15]:

$$
\int \boldsymbol{h}(\boldsymbol{x}) \phi(\boldsymbol{x} ; \mathbf{0}, \boldsymbol{I}) \mathrm{d} \boldsymbol{x} \approx \frac{1}{2 n} \sum_{i=1}^{n}\left[\boldsymbol{h}\left(\sqrt{n} \boldsymbol{e}_{i}\right)+\boldsymbol{h}\left(-\sqrt{n} \boldsymbol{e}_{i}\right)\right] .
$$

Remark: The third-degree cubature rule is identical to the unscented transformation with $\kappa=0$.

To construct the fifth-degree cubature rule, the Mysovskikh's method [29] and the moment matching method [16] are used to provide the fifth-degree spherical rule and radial rule, respectively. The final fifth-degree cubature rule is given by

$$
\begin{aligned}
\int \boldsymbol{h}(\boldsymbol{x}) \phi(\boldsymbol{x} ; \mathbf{0}, \boldsymbol{I}) \mathrm{d} \boldsymbol{x} \approx & \frac{2}{n+2} \boldsymbol{h}(\mathbf{0})+ \\
& +\frac{n^{2}(7-n)}{2(n+1)^{2}(n+2)^{2}} \sum_{i=1}^{n+1}\left[\boldsymbol{h}\left(\sqrt{n+2} \boldsymbol{s}_{1}^{(i)}\right)\right. \\
& \left.+\boldsymbol{h}\left(-\sqrt{n+2} \boldsymbol{s}_{1}^{(i)}\right)\right] \\
& +\frac{2(n-1)^{2}}{(n+1)^{2}(n+2)^{2}} \sum_{i=1}^{n(n+1) / 2}\left[\boldsymbol{h}\left(\sqrt{n+2} \boldsymbol{s}_{2}^{(i)}\right)\right. \\
& \left.+\boldsymbol{h}\left(-\sqrt{n+2} \boldsymbol{s}_{2}^{(i)}\right)\right],
\end{aligned}
$$

where the point $s_{1}^{(i)}$ is given by

$$
\boldsymbol{s}_{1}^{(i)}=\left[p_{1}^{(i)}, p_{2}^{(i)}, \cdots, p_{n}^{(i)}\right], \quad i=1,2, \cdots, n+1,
$$

with

$$
p_{j}^{(i)}= \begin{cases}-\sqrt{\frac{n+1}{n(n-j+2)(n-j+1)}}, & j<i, \\ \sqrt{\frac{(n+1)(n-i+1)}{n(n-i+2)},} & j=i, \\ 0, & j>i .\end{cases}
$$

Moreover, the set of points $\left\{\boldsymbol{s}_{2}^{(i)}\right\}$ is given by

$$
\begin{gathered}
\left\{s_{2}^{(i)}\right\}=\left\{\sqrt{\frac{n}{2(n-1)}}\left(s_{1}^{(k)}+s_{1}^{(l)}\right): k<l, k, l=1,\right. \\
2, \cdots, n+1\} .
\end{gathered}
$$

3.3 Augmented state-space model for network inference In the state-space model for gene regulatory networks described in Section 3.2, the underlying network structure is characterized by the $n \times n$ regulatory coefficient matrix $\boldsymbol{A}$ in (2) and the parameters $\boldsymbol{\mu}=\left[\mu_{1}, \ldots, \mu_{n}\right]$ in (4). The problem of network inference then becomes to estimate $A$ and $\boldsymbol{\mu}$. To do that, we incorporate the unknown parameters $\boldsymbol{A}$ and $\boldsymbol{\mu}$ into the state vector to obtain an augmented state-space model, and then apply the point-based Gaussian approximation filters to estimate the space vector and thereby obtaining the estimates of $\boldsymbol{A}$ and $\boldsymbol{\mu}$.

Specifically, we denote $\boldsymbol{\theta}=\left[a_{11}, a_{12}, \cdots, a_{1 n}, \cdots\right.$, $\left.a_{n n}, \mu_{1}, \cdots, \mu_{n}\right]^{T}$ and the augmented state vector $\overline{\boldsymbol{x}}_{k}=$ $\left[\boldsymbol{x}_{k}^{T}, \boldsymbol{\theta}^{T}\right]^{T}$. Then, the augmented state equation can be
written as

$$
\overline{\boldsymbol{x}}_{k}=\overline{\boldsymbol{f}}\left(\overline{\boldsymbol{x}}_{k-1}\right)+\overline{\boldsymbol{v}}_{k}=\left[\begin{array}{c}
\boldsymbol{A}_{k-1} \boldsymbol{g}_{k-1}\left(\boldsymbol{x}_{k-1}\right) \\
\boldsymbol{\theta}_{k-1}
\end{array}\right]+\left[\begin{array}{c}
\boldsymbol{v}_{k-1} \\
\mathbf{0}
\end{array}\right] .
$$

Note that $\boldsymbol{A}_{k-1}$ and $\boldsymbol{g}_{k-1}$ can be obtained from $\boldsymbol{\theta}_{k-1}$, and $\overline{\boldsymbol{v}}_{k} \sim \mathcal{N}\left(\mathbf{0}, \overline{\boldsymbol{Q}}_{k}\right)$ with $\overline{\boldsymbol{Q}}_{k}=\operatorname{diag}\left(\left[\begin{array}{ll}\boldsymbol{Q}_{k} & \boldsymbol{O}_{n^{2}+n}\end{array}\right]\right)$, where $\boldsymbol{O}_{m}$ denotes an $m \times m$ all-zero matrix.

In the remainder of this paper, we assume that the noisy gene expression levels are observed. Therefore, the augmented measurement equation becomes

$$
\boldsymbol{y}_{k}=\boldsymbol{h}\left(\overline{\boldsymbol{x}}_{k}\right)+\boldsymbol{n}_{k}=\boldsymbol{B} \overline{\boldsymbol{x}}_{k}+\boldsymbol{n}_{k},
$$

where $\boldsymbol{B}=\left[\boldsymbol{I}_{n}, \boldsymbol{O}_{n \times\left(n^{2}+n\right)}\right], \boldsymbol{O}_{n \times\left(n^{2}+n\right)}$ denotes an $n \times$ $\left(n^{2}+n\right)$ all zeros matrix.

The point-based Gaussian approximation filters can then be used to obtain the estimate of the augmented state, $\hat{\bar{x}}_{k}$, from which the estimates of the unknown network parameters, i.e., $\hat{A}$ and $\hat{\mu}$ can then be obtained.

Note that since the measurement Equation (41) is linear, the filtering Equations $(10,11)$ become

$$
\begin{aligned}
\hat{\overline{\boldsymbol{x}}}_{k \mid k} & =\hat{\overline{\boldsymbol{x}}}_{k \mid k-1}+\boldsymbol{L}_{k}\left(\boldsymbol{y}_{k}-\boldsymbol{B} \hat{\overline{\boldsymbol{x}}}_{k \mid k-1}\right), \\
\text { and } \boldsymbol{P}_{k \mid k} & =\boldsymbol{P}_{k \mid k-1}-\boldsymbol{L}_{k} \boldsymbol{B} \boldsymbol{P}_{k \mid k-1} \\
\text { with } \boldsymbol{L}_{k} & =\boldsymbol{P}_{k \mid k-1} \boldsymbol{B}^{T}\left(\boldsymbol{R}_{k}+\boldsymbol{B} \boldsymbol{P}_{k \mid k-1} \boldsymbol{B}^{T}\right)^{-1},
\end{aligned}
$$

which are the same as the filtering updates for Kalman filters.

\section{Incorporating prior information}

In practice, some prior knowledge on the underlying GRN is typically available. In this section, we outline approaches to incorporating such prior knowledge into the point-based Gaussian approximation filters for network inference. In particular, we consider two types of prior information, namely, sparsity constraints and range constraints on the network. For networks with sparsity constraints, we incorporate an iterative thresholding procedure into the Gaussian approximation filters. 
And to accommodate range constraints, we employ PDFtruncated Gaussian approximation filters.

\subsection{Optimization-based approach for sparsity constraints 4.1.1 The optimization formulations}

Note that under the Gaussian assumption, the state estimation $\hat{\overline{\boldsymbol{x}}}_{k \mid k}$ of the Kalman filter is equivalently given by the solution to the following optimization problem [30,31]

$$
\begin{aligned}
\hat{\overline{\boldsymbol{x}}}_{k \mid k}= & \underset{\overline{\boldsymbol{x}}}{\arg \min } J(\overline{\boldsymbol{x}}), \\
\text { with } J(\overline{\boldsymbol{x}}) \triangleq & \left(\boldsymbol{y}_{k}-\boldsymbol{h}(\overline{\boldsymbol{x}})\right)^{T} \boldsymbol{R}_{k}^{-1}\left(\boldsymbol{y}_{k}-\boldsymbol{h}(\overline{\boldsymbol{x}})\right) \\
& +\left(\overline{\boldsymbol{x}}-\hat{\overline{\boldsymbol{x}}}_{k \mid k-1}\right)^{T} \boldsymbol{P}_{k \mid k-1}^{-1}\left(\overline{\boldsymbol{x}}-\hat{\overline{\boldsymbol{x}}}_{k \mid k-1}\right) .
\end{aligned}
$$

To incorporate the prior information of the GRN, (46) is modified as

$$
\tilde{J}(\overline{\boldsymbol{x}})=J(\overline{\boldsymbol{x}})+\lambda J_{p}(\overline{\boldsymbol{x}}),
$$

where $J_{p}(\overline{\boldsymbol{x}})$ is a penalty function associated with the prior information and $\lambda$ is a tunable parameter that regulates the tightness of the penalty.

For example, in gene regulatory networks, each gene only interacts with a few genes [20]. To capture such a sparsity constraint, a Laplace prior distribution can be used for the connection coefficient matrix $A$, i.e.,

$$
p(\boldsymbol{A})=(\lambda / 2)^{n^{2}} \exp \left(-\lambda \sum_{i=1}^{n} \sum_{j=1}^{n}\left|a_{i j}\right|\right) .
$$

Therefore, in this case, $J_{p}(\overline{\boldsymbol{x}})=-\log p(\boldsymbol{A})=c_{1}\|\boldsymbol{A}\|_{1}+c_{2}$ where $c_{1}$ and $c_{2}$ are constants. And, (47) can be rewritten as

$$
\tilde{J}(\overline{\boldsymbol{x}})=J(\overline{\boldsymbol{x}})+\lambda\|\boldsymbol{A}\|_{1} .
$$

Note that (49) can also be interpreted as the result of applying the least squares shrinkage selection operator (LASSO) to (47). The LASSO adds an $L_{1}$-norm constraint to the GRN so that the regulatory coefficient matrix $A$ tends to be sparse with many zero elements.

As another example, if some known regulatory relationship exists, then it should be taken into account to improve the estimation accuracy. Specifically, define an $n \times n$ indicator matrix $\boldsymbol{E}=\left[e_{i, j}\right]$ where $e_{i j}=1$ indicates that there is a lack of regulation from gene $j$ to gene $i$. Then, similar to the use of LASSO, a penalty on $a_{i j}$ should incur if $e_{i j}=1$. Thus, (47) can be rewritten as

$$
\tilde{J}(\overline{\boldsymbol{x}})=J(\overline{\boldsymbol{x}})+\lambda\|\boldsymbol{E} \circ \boldsymbol{A}\|_{1} .
$$

Note that as in [20], here we do not force $a_{i j}=0$ corresponding to $e_{i j}=1$ but rather use an $L_{1}$-norm penalty. The advantage of such an approach is that it allows the algorithm to pick different structures but more likely to pick the edges without penalties. 'o' denotes the entrywise product operation of two matrices.

\subsubsection{Iterative thresholding algorithm}

Solving the optimization problems in (49) and (50) is not straightforward since $|a|$ is non-differentiable at $a=0$. In the following, an efficient solver called the iterative thresholding algorithm is introduced.

For convenience, we consider a general optimization problem of the form

$$
\underset{\bar{x}}{\arg \min } J(\overline{\boldsymbol{x}})=L(\overline{\boldsymbol{x}})+\|\lambda \circ \overline{\boldsymbol{x}}\|_{1},
$$

where $\lambda=\left[\lambda_{1}, \lambda_{2}, \cdots, \lambda_{n^{2}+2 n}\right]^{T}$ and $L(\overline{\boldsymbol{x}})$ is a smooth function. Note that if $\lambda=\left[\mathbf{0}_{1 \times n}, \lambda \times \mathbf{1}_{1 \times n^{2}}, \mathbf{0}_{1 \times n}\right]^{T}$, then (51) becomes (49); and if $\lambda=\left[\mathbf{0}_{1 \times n}, \lambda \times \underline{\hat{\boldsymbol{\theta}}}, \mathbf{0}_{1 \times n}\right]^{T}$, then (51) becomes (50). Note that $\underline{\hat{\boldsymbol{\theta}}}=\left[e_{11}, e_{12}, \cdots, e_{1 n}, \cdots, e_{n n}\right]^{T}$.

The solution to (51) can be iteratively obtained by solving a sequence of optimization problems. As in Newton's method, the Taylor series expansion of $L(\overline{\boldsymbol{x}})$ around the solution $\overline{\boldsymbol{x}}^{t}$ at the $t$-th iteration is given by

$$
L\left(\overline{\boldsymbol{x}}^{t}+\Delta \overline{\boldsymbol{x}}\right) \cong L\left(\overline{\boldsymbol{x}}^{t}\right)+\Delta \overline{\boldsymbol{x}}^{T} \nabla L\left(\overline{\boldsymbol{x}}^{t}\right)+\frac{\alpha_{t}}{2}\|\Delta \overline{\boldsymbol{x}}\|_{2}^{2},
$$

where $\nabla L$ is the gradient of $L$ and $\alpha_{t}$ is such that $\alpha_{t} \boldsymbol{I}$ mimics the Hessian $\nabla^{2} L$. Then, $\bar{x}^{t+1}$ is given by [32]

$$
\overline{\boldsymbol{x}}^{t+1}=\underset{\boldsymbol{z}}{\arg \min }\left(\boldsymbol{z}-\overline{\boldsymbol{x}}^{t}\right)^{T} \nabla L\left(\overline{\boldsymbol{x}}^{t}\right)+\frac{\alpha_{t}}{2}\left\|\boldsymbol{z}-\overline{\boldsymbol{x}}^{t}\right\|_{2}^{2}+\|\lambda \circ \boldsymbol{z}\|_{1} .
$$

The equivalent form of (53) is given by [32]

$$
\begin{aligned}
\overline{\boldsymbol{x}}^{t+1} & =\underset{\boldsymbol{z}}{\arg \min } \frac{1}{2}\left\|\boldsymbol{z}-\boldsymbol{u}^{t}\right\|_{2}^{2}+\frac{1}{\alpha_{t}}\|\lambda \circ \boldsymbol{z}\|_{1}, \\
\text { with } \boldsymbol{u}^{t} & =\overline{\boldsymbol{x}}^{t}-\frac{1}{\alpha_{t}} \nabla L\left(\overline{\boldsymbol{x}}^{t}\right), \\
\alpha_{t} & \approx \frac{\left(\boldsymbol{s}^{t}\right)^{T} \boldsymbol{r}^{t}}{\left\|\boldsymbol{s}^{t}\right\|^{2}} \\
\boldsymbol{s}^{t} & =\overline{\boldsymbol{x}}^{t}-\overline{\boldsymbol{x}}^{t-1} \\
\boldsymbol{r}^{t} & =\nabla L\left(\overline{\boldsymbol{x}}^{t}\right)-\nabla L\left(\overline{\boldsymbol{x}}^{t-1}\right) .
\end{aligned}
$$

The solution to (54) is given by [32] $\overline{\boldsymbol{x}}^{t+1}=\eta^{S}\left(\boldsymbol{u}^{t}, \frac{\lambda}{\alpha_{t}}\right)$, where

$$
\eta^{S}(\boldsymbol{u}, \boldsymbol{a})=\operatorname{sign}(\boldsymbol{u}) \max \{|\boldsymbol{u}|-\boldsymbol{a}, \mathbf{0}\}
$$

is the soft thresholding function with $\operatorname{sign}(\boldsymbol{u})$ and $\max \{|\boldsymbol{u}|-\boldsymbol{a}, \mathbf{0}\}$ being component-wise operators. by

Finally, the iterative procedure for solving (51) is given

$$
\overline{\boldsymbol{x}}^{t+1}=\operatorname{sign}\left(\overline{\boldsymbol{x}}^{t}-\frac{1}{\alpha_{t}} \nabla L\left(\overline{\boldsymbol{x}}^{t}\right)\right) \max \left\{\left|\overline{\boldsymbol{x}}^{t}-\frac{1}{\alpha_{t}} \nabla L\left(\overline{\boldsymbol{x}}^{t}\right)\right|-\frac{\lambda}{\alpha_{t}}, \mathbf{0}\right\} .
$$


And the iteration stops when the following condition is met

$$
\frac{\left|J\left(\overline{\boldsymbol{x}}^{t}\right)-J\left(\overline{\boldsymbol{x}}^{t-1}\right)\right|}{\left|J\left(\overline{\boldsymbol{x}}^{t-1}\right)\right|} \leq \epsilon,
$$

where $\epsilon$ is a given small number.

\subsection{PDF truncation method for range constraints}

If the range constraints on the regulatory coefficients are available, the inference accuracy can be improved by enforcing the constraints in the Gaussian approximation filters.

In particular, assume that we impose the following range constraints on the state vector $\bar{x}$

$$
c \leq \bar{x} \leq d
$$

The PDF truncation method [31] can be employed to incorporate the above range constraint into the Gaussian approximation filters, by converting the updated mean $\hat{\overline{\boldsymbol{x}}}_{k \mid k}$ and covariance $\boldsymbol{P}_{k \mid k}$ to the pseudo mean $\hat{\bar{x}}_{k \mid k}^{t}$ and covariance $\boldsymbol{P}_{k \mid k}^{t}$ which are then used in the next prediction and filtering steps.

We next briefly outline the PDF truncation procedure. We use $\hat{\overline{\boldsymbol{x}}}_{k \mid k, i}^{t}$ and $\boldsymbol{P}_{k \mid k, i}^{t}$ to denote the mean and covariance after the first $i$ constraints have been enforced. Initially, we set $\hat{\overline{\boldsymbol{x}}}_{k \mid k, 0}^{t}=\hat{\overline{\boldsymbol{x}}}_{k \mid k}$ and $\boldsymbol{P}_{k \mid k, 0}^{t}=\boldsymbol{P}_{k \mid k}$. Consider the following transformation

$$
z_{k, i}=\mathcal{G}_{i} \mathcal{D}_{i}^{-1 / 2} \mathcal{S}_{i}^{T}\left(\overline{\boldsymbol{x}}_{k}-\hat{\overline{\boldsymbol{x}}}_{k \mid k, i}^{t}\right)
$$

where $\mathcal{S}_{i}$ and $\mathcal{D}_{i}$ are obtained from the Jordan canonical decomposition $\mathcal{S}_{i} \mathcal{D}_{i} \mathcal{S}_{i}^{T}=\boldsymbol{P}_{k \mid k, i}^{t}$ and $\mathcal{G}_{i}$ is obtained by using the Gram-Schmidt orthogonalization and it satisfies [33]

$$
\mathcal{G}_{i} \mathcal{D}_{i}^{1 / 2} \mathcal{S}_{i}^{T} \boldsymbol{e}_{i}=\left[\left(\boldsymbol{e}_{i}^{T} \boldsymbol{P}_{k \mid k, i}^{t} \boldsymbol{e}_{i}\right)^{1 / 2}, 0, \cdots, 0\right]
$$

Then, the upper bound $\boldsymbol{e}_{i}^{T} \overline{\boldsymbol{x}} \leq d_{i}$ is transformed to [33]

$$
[1,0, \cdots, 0] \boldsymbol{z}_{k, i} \leq \frac{d_{i}-\boldsymbol{e}_{i}^{T} \hat{\overline{\boldsymbol{x}}}_{k \mid k, i}^{t}}{\left(\boldsymbol{e}_{i}^{T} \boldsymbol{P}_{k \mid k, i}^{t} \boldsymbol{e}_{i}\right)^{1 / 2}} \triangleq \tilde{d}_{i}
$$

Similarly, the lower bound $\boldsymbol{e}_{i}^{T} \overline{\boldsymbol{x}} \geq c_{i}$ is transformed to

$$
[1,0, \cdots, 0] \boldsymbol{z}_{k, i} \geq \frac{c_{i}-\boldsymbol{e}_{i}^{T} \hat{\bar{x}}_{k \mid k, i}^{t}}{\left(\boldsymbol{e}_{i}^{T} \boldsymbol{P}_{k \mid k, i}^{t} \boldsymbol{e}_{i}\right)^{1 / 2}} \triangleq \tilde{c}_{i} .
$$

The constraint requires that the first element of $\boldsymbol{z}_{k, i}$ lies between $\tilde{c}_{i}$ and $\tilde{d}_{i}$. Hence, only the truncated PDF of the first element of $z_{k, i}$ is considered and it is given by [33]

$$
\begin{aligned}
f(z) & =\alpha_{i} \exp \left(-z^{2} / 2\right), \\
\text { with } \quad \alpha_{i} & =\frac{\sqrt{2}}{\sqrt{\pi}\left[\operatorname{erf}\left(\tilde{d}_{i} / \sqrt{2}\right)-\operatorname{erf}\left(\tilde{c}_{i} / \sqrt{2}\right)\right]} .
\end{aligned}
$$

Then, the mean and variance of the first element of $\boldsymbol{z}_{k, i}$ after imposing the $i$-th constraint are given respectively by

$$
\begin{aligned}
\mu_{i}= & \int_{\tilde{c}_{i}}^{\tilde{d}_{i}} z f(z) \mathrm{d} z=\alpha_{i}\left[\exp \left(-\tilde{c}_{i}^{2} / 2\right)-\exp \left(-\tilde{d}_{i}^{2} / 2\right)\right] \\
\sigma_{i}^{2}= & \int_{\tilde{c}_{i}}^{\tilde{d}_{i}}\left(z-\mu_{i}\right)^{2} f(z) \mathrm{d} z \\
= & \alpha_{i}\left[\exp \left(-\tilde{c}_{i}^{2} / 2\right)\left(\tilde{c}_{i}-2 \mu_{i}\right)\right. \\
& \left.-\exp \left(-\tilde{d}_{i}^{2} / 2\right)\left(\tilde{d}_{i}-2 \mu_{i}\right)\right]+\mu_{i}^{2}+1
\end{aligned}
$$

Thus, the mean and covariance of the transformed state vector after imposing the $i$-th constraint are given respectively by

$$
\begin{aligned}
\overline{\boldsymbol{z}}_{k, i} & =\left[\mu_{i}, 0, \cdots, 0\right]^{T}, \\
\boldsymbol{Q}_{k, i} & =\operatorname{diag}\left(\left[\sigma_{i}^{2}, 1, \cdots, 1\right]\right) .
\end{aligned}
$$

\begin{tabular}{|c|c|c|c|c|c|c|c|c|c|}
\hline \multirow[b]{2}{*}{ Filters } & \multicolumn{3}{|c|}{ True positive rate } & \multicolumn{3}{|c|}{ False positive rate } & \multicolumn{3}{|c|}{ Positive predictive rate } \\
\hline & Min & $\operatorname{Max}$ & Avg & Min & Max & Avg & Min & Max & Avg \\
\hline $\operatorname{UKF}(\kappa=-5)$ & 0.7576 & 0.9355 & 0.8472 & 0.5000 & 0.7647 & 0.5955 & 0.5094 & 0.6279 & 0.5824 \\
\hline $\operatorname{UKF}(\kappa=-2)$ & 0.7576 & 0.9355 & 0.8406 & 0.5161 & 0.7647 & 0.5933 & 0.5094 & 0.6279 & 0.5825 \\
\hline $\operatorname{UKF}(\kappa=0)$ & 0.7576 & 0.9375 & 0.8426 & 0.5161 & 0.7647 & 0.5918 & 0.5094 & 0.6364 & 0.5840 \\
\hline $\operatorname{UKF}(\kappa=2)$ & 0.7576 & 0.9375 & 0.8407 & 0.5152 & 0.7353 & 0.5895 & 0.5098 & 0.6279 & 0.5841 \\
\hline $\operatorname{UKF}(\kappa=5)$ & 0.7576 & 0.9063 & 0.8394 & 0.5161 & 0.7353 & 0.5933 & 0.5192 & 0.6279 & 0.5821 \\
\hline
\end{tabular}

By taking the inverse transform of (63), we then get

$$
\begin{aligned}
& \hat{\overline{\boldsymbol{x}}}_{k \mid k, i+1}^{t}=\mathcal{S}_{i} \mathcal{D}_{i}^{1 / 2} \mathcal{G}_{i}^{T} \overline{\boldsymbol{z}}_{k, i}+\hat{\overline{\boldsymbol{x}}}_{k \mid k, i}^{t}, \\
& \boldsymbol{P}_{k \mid k, i+1}^{t}=\mathcal{S}_{i} \mathcal{D}_{i}^{1 / 2} \mathcal{G}_{i}^{T} \boldsymbol{Q}_{k, i} \mathcal{G}_{i} \mathcal{D}_{i}^{1 / 2} \mathcal{S}_{i}^{T} .
\end{aligned}
$$

After imposing all $n$ constraints, the final constrained state estimate and covariance at time $k$ are given respectively by $\hat{\bar{x}}_{k \mid k}^{t} \triangleq \hat{\overline{\boldsymbol{x}}}_{k \mid k, n}^{t}$ and $\boldsymbol{P}_{k \mid k}^{t} \triangleq \boldsymbol{P}_{k \mid k, n}^{t}$.

\section{Numerical results}

\subsection{Synthetic network}

In this section, a synthetic network that contains eight genes is used to test the performance of the EKF, the

Table 1 Comparison of UKF with different $\kappa$ 
Table 2 Comparison of different filters

\begin{tabular}{|c|c|c|c|c|c|c|c|c|c|c|c|c|}
\hline \multirow[b]{2}{*}{ Filters } & \multicolumn{3}{|c|}{ True positives \# } & \multicolumn{3}{|c|}{ False positives \# } & \multicolumn{3}{|c|}{ True negatives \# } & \multicolumn{3}{|c|}{ False negatives \# } \\
\hline & Min & Max & Avg & Min & Max & Avg & Min & Max & Avg & Min & Max & Avg \\
\hline EKF & 2 & 17 & 10.60 & 23 & 44 & 36.4 & 2 & 15 & 7.08 & 2 & 24 & 9.92 \\
\hline UKF & 25 & 29 & 26.80 & 16 & 26 & 19.28 & 8 & 16 & 13.06 & 2 & 8 & 4.86 \\
\hline $\mathrm{CKF}_{3}$ & 25 & 30 & 26.74 & 16 & 26 & 19.10 & 8 & 15 & 13.14 & 2 & 8 & 5.02 \\
\hline \multirow[t]{2}{*}{$\mathrm{CKF}_{5}$} & 25 & 29 & 26.64 & 16 & 26 & 19.24 & 8 & 16 & 13.08 & 1 & 8 & 5.04 \\
\hline & \multicolumn{5}{|c|}{ True positive rate } & & \multicolumn{3}{|c|}{ False positive rate } & \multicolumn{3}{|c|}{ Positive predictive rate } \\
\hline Filters & \multicolumn{2}{|r|}{ Min } & \multicolumn{2}{|c|}{ Max } & \multicolumn{2}{|l|}{ Avg } & Min & Max & Avg & Min & Max & Avg \\
\hline EKF & \multicolumn{2}{|r|}{0.0769} & \multicolumn{2}{|c|}{0.8667} & \multicolumn{2}{|l|}{0.5224} & 0.6053 & 0.9545 & 0.8358 & 0.0800 & 0.3208 & 0.2231 \\
\hline UKF & \multicolumn{2}{|r|}{0.7576} & \multicolumn{2}{|c|}{0.9355} & \multicolumn{2}{|l|}{0.8472} & 0.5 & 0.7576 & 0.5955 & 0.5094 & 0.6279 & 0.5824 \\
\hline $\mathrm{CKF}_{3}$ & \multicolumn{2}{|r|}{0.7576} & \multicolumn{2}{|c|}{0.9375} & \multicolumn{2}{|l|}{0.8426} & 0.5161 & 0.7647 & 0.5918 & 0.5094 & 0.6364 & 0.5840 \\
\hline$\overline{\mathrm{CKF}_{5}}$ & \multicolumn{2}{|r|}{0.7576} & \multicolumn{2}{|c|}{0.9667} & \multicolumn{2}{|l|}{0.8417} & 0.5000 & 0.7647 & 0.5946 & 0.5094 & 0.6279 & 0.5814 \\
\hline
\end{tabular}


Table 3 Inferred results of the conventional filter and filters incorporating the prior information

\begin{tabular}{|c|c|c|c|c|c|c|c|c|c|c|c|c|}
\hline \multirow[b]{2}{*}{ Filters } & \multicolumn{3}{|c|}{ True positives \# } & \multicolumn{3}{|c|}{ False positives \# } & \multicolumn{3}{|c|}{ True negatives \# } & \multicolumn{3}{|c|}{ False negatives \# } \\
\hline & Min & Max & Avg & Min & Max & Avg & Min & Max & Avg & Min & Max & Avg \\
\hline UKF & 25 & 29 & 26.80 & 16 & 26 & 19.28 & 8 & 16 & 13.06 & 2 & 8 & 4.86 \\
\hline $\mathrm{UKF}_{p 1}$ & 25 & 29 & 27.34 & 14 & 19 & 16.52 & 13 & 18 & 15.72 & 2 & 8 & 4.42 \\
\hline $\mathrm{UKF}_{p 2}$ & 23 & 26 & 24.16 & 13 & 16 & 13.86 & 16 & 18 & 17.20 & 7 & 10 & 8.78 \\
\hline \multirow[t]{2}{*}{$\mathrm{UKF}_{p 3}$} & 25 & 29 & 26.70 & 12 & 24 & 17.50 & 9 & 19 & 14.50 & 3 & 8 & 5.30 \\
\hline & \multicolumn{6}{|c|}{ True positive rate } & \multicolumn{3}{|c|}{ False positive rate } & \multicolumn{3}{|c|}{ Positive predictive rate } \\
\hline Filters & \multicolumn{2}{|r|}{ Min } & \multicolumn{2}{|c|}{ Max } & \multicolumn{2}{|c|}{ Avg } & Min & Max & Avg & Min & Max & Avg \\
\hline UKF & \multicolumn{2}{|r|}{0.7576} & \multicolumn{2}{|c|}{0.9355} & \multicolumn{2}{|c|}{0.8472} & 0.5 & 0.7647 & 0.5955 & 0.5094 & 0.6279 & 0.5824 \\
\hline $\mathrm{UKF}_{p 1}$ & \multicolumn{2}{|r|}{0.7576} & \multicolumn{2}{|c|}{0.9355} & \multicolumn{2}{|c|}{0.8614} & 0.4375 & 0.5935 & 0.5121 & 0.5778 & 0.6744 & 0.6239 \\
\hline $\mathrm{UKF}_{p 2}$ & \multicolumn{2}{|r|}{0.6970} & \multicolumn{2}{|c|}{0.7879} & \multicolumn{2}{|c|}{0.7335} & 0.4194 & 0.5000 & 0.4462 & 0.5897 & 0.6667 & 0.6355 \\
\hline $\mathrm{UKF}_{p 3}$ & \multicolumn{2}{|r|}{0.7576} & \multicolumn{2}{|c|}{0.9063} & \multicolumn{2}{|c|}{0.8348} & 0.3871 & 0.7273 & 0.5463 & 0.5294 & 0.6923 & 0.6049 \\
\hline
\end{tabular}


Table 4 Comparison of $\mathrm{UKF}_{p 1}$ using different $\lambda$

\begin{tabular}{|c|c|c|c|c|c|c|c|c|c|}
\hline \multirow[b]{2}{*}{ Filters } & \multicolumn{3}{|c|}{ True positive rate } & \multicolumn{3}{|c|}{ False positive rate } & \multicolumn{3}{|c|}{ Positive predictive rate } \\
\hline & Min & Max & Avg & Min & Max & Avg & Min & Max & Avg \\
\hline $\mathrm{UKF}_{p 1}(\lambda=0.1)$ & 0.7576 & 0.9355 & 0.8484 & 0.5000 & 0.7647 & 0.5900 & 0.5094 & 0.6279 & 0.5850 \\
\hline $\mathrm{UKF}_{p 1}(\lambda=0.5)$ & 0.7576 & 0.9677 & 0.8535 & 0.4688 & 0.7647 & 0.5696 & 0.5094 & 0.6512 & 0.5948 \\
\hline $\mathrm{UKF}_{p 1}(\lambda=1)$ & 0.7576 & 0.9355 & 0.8614 & 0.4375 & 0.5935 & 0.5121 & 0.5778 & 0.6744 & 0.6239 \\
\hline $\mathrm{UKF}_{p 1}(\lambda=5)$ & 0.7500 & 0.9355 & 0.8439 & 0.3548 & 0.5455 & 0.4672 & 0.5814 & 0.7105 & 0.6456 \\
\hline $\operatorname{UKF}_{p 1}(\lambda=10)$ & 0.7273 & 0.9063 & 0.8217 & 0.3226 & 0.4848 & 0.4156 & 0.6190 & 0.7368 & 0.6695 \\
\hline
\end{tabular}

UKF, the $\mathrm{CKF}_{3}$, the $\mathrm{CKF}_{5}$, and their corresponding filters incorporating the prior information. Forty data points are collected to infer the structure of the network. The system noise and measurement noise are assumed to be Gaussian distributed with means $\mathbf{0}$ and covariances $\overline{\boldsymbol{Q}}_{k}=$ $\operatorname{diag}\left(\left[\begin{array}{ll}0.01 \boldsymbol{I}_{8} & \boldsymbol{O}_{72}\end{array}\right]\right)$ and $\boldsymbol{R}_{k}=0.01 \boldsymbol{I}_{8}$, respectively. The connection coefficient matrix is given by

$$
\boldsymbol{A}=\left(\begin{array}{cccccccc}
0 & 0 & 0 & 0 & 0 & 0 & 2.4 & 3.2 \\
0 & 0 & 0 & 4.1 & 0 & -2.4 & 0 & 4.1 \\
-5.0 & 2.1 & -1.5 & 0 & 4.5 & 0 & 2.1 & 0 \\
0 & 1.3 & 2.5 & -3.7 & 1.8 & 0 & 0 & -3.1 \\
0 & 0 & 0 & -2.6 & -3.2 & 0 & -1 & 4 \\
-1.5 & -1.8 & 0 & 3.4 & 1.4 & 1.1 & 0 & 1.7 \\
-1.8 & 0 & 0 & -3 & 1.1 & 2.4 & 0 & 0 \\
-1.3 & 0 & -1 & 0 & 2.1 & 0 & 0 & 2.2
\end{array}\right)
$$

and $\mu_{i}=2, i=1, \cdots, 8$. For the filter, each coefficient in $\hat{A}$ is initialized from a Gaussian distribution with mean 0 and variance 0.2 . Moreover, the coefficient $\mu_{i}$ is initialized from a Gaussian distribution with mean 1.5 and variance 0.2 . The system state is initialized using the first measurement.

The metric used to evaluate the inferred GRN is the true positive rate (TPR), the false positive rate (FPR), and the positive predictive value (PPV). They are given by [34]

$$
\begin{aligned}
& \mathrm{TPR}=\frac{\mathrm{TP \#}}{\mathrm{TP \#}+\mathrm{FN \#},} \\
& \mathrm{FPR}=\frac{\mathrm{FP \#}}{\mathrm{FP \#}+\mathrm{TN \#}}, \\
& \mathrm{PPV}=\frac{\mathrm{TP \#}}{\mathrm{TP \#}+\mathrm{FP \#}},
\end{aligned}
$$

where the number of true positives (TP\#) denotes the number of links correctly predicted by the inference algorithm; the number of false positives (FP\#) denotes the number of incorrectly predicted links; the number of true negatives (TN\#) denotes the number of correctly predicted nonlinks; and the number of false negatives (FN\#) denotes the number of missed links by the inference algorithm [8].

\subsubsection{Comparison of the EKF with point-based Gaussian approximation filters}

The UKF with different parameter $\kappa$ is tested. The simulation results based on 50 Monte Carlo runs are shown in Table 1 . It can be seen that UKFs with $\kappa=0,2,5$ have slight better performance than UKFs with $\kappa=-5,-2$. One possible reason is that the weights of all sigma points used in the UKF are all positive when $\kappa \geq 0$. In general, all positive weights will guarantee better stability of the filtering algorithm. However, it should be emphasized that, in this specific example, there is no big difference between UKFs with different $\kappa$. In addition, the objective of this paper was to investigate the proposed filter incorporating the prior information. Hence, the UKF is used to denote UKF with $\kappa=3-n$ and compare with the filters incorporating the prior information.

The inference results of the EKF, the UKF, the $\mathrm{CKF}_{3}$, and the $\mathrm{CKF}_{5}$ are summarized in Table 2, all results are based on 50 Monte Carlo runs. It can be seen that all point-based Gaussian approximation filters have better performance than the EKF since the average(avg) FPR is

\begin{tabular}{|c|c|c|c|c|c|c|c|c|c|}
\hline \multirow[b]{2}{*}{ Filters } & \multicolumn{3}{|c|}{ True positive rate } & \multicolumn{3}{|c|}{ False positive rate } & \multicolumn{3}{|c|}{ Positive predictive rate } \\
\hline & Min & Max & Avg & Min & $\operatorname{Max}$ & Avg & Min & Max & Avg \\
\hline $\operatorname{UKF}_{\tilde{p} 1}(\lambda=0.1)$ & 0.7576 & 0.9677 & 0.8484 & 0.4688 & 0.7647 & 0.5713 & 0.5094 & 0.6512 & 0.5929 \\
\hline $\mathrm{UKF}_{\tilde{p} 1}(\lambda=0.5)$ & 0.7576 & 0.9333 & 0.8468 & 0.4516 & 0.7059 & 0.5422 & 0.5385 & 0.6512 & 0.6057 \\
\hline $\mathrm{UKF}_{\tilde{p} 1}(\lambda=1)$ & 0.7500 & 0.9032 & 0.8221 & 0.3750 & 0.5758 & 0.4953 & 0.5814 & 0.6842 & 0.6257 \\
\hline $\mathrm{UKF}_{\tilde{p} 1}(\lambda=5)$ & 0.7273 & 0.8750 & 0.8220 & 0.3548 & 0.5000 & 0.4169 & 0.6098 & 0.7179 & 0.6684 \\
\hline $\mathrm{UKF}_{\tilde{p} 1}(\lambda=10)$ & 0.7500 & 0.8750 & 0.8214 & 0.3226 & 0.5000 & 0.4143 & 0.6098 & 0.7368 & 0.6696 \\
\hline
\end{tabular}
lower and the average TPR and precision are higher than that of the EKF. Although the CKFs exhibit slightly better

Table 5 Effect of strength of the links using different $\lambda$ 
Table 6 Effect of false prior information using different $\lambda$

\begin{tabular}{|c|c|c|c|c|c|c|c|c|c|}
\hline \multirow[b]{2}{*}{ Filters } & \multicolumn{3}{|c|}{ True positive rate } & \multicolumn{3}{|c|}{ False positive rate } & \multicolumn{3}{|c|}{ Positive predictive rate } \\
\hline & Min & Max & Avg & Min & Max & Avg & Min & Max & Avg \\
\hline $\mathrm{UKF}_{\bar{p} 1}(\lambda=0.1)$ & 0.7576 & 0.9667 & 0.8491 & 0.5000 & 0.7647 & 0.5933 & 0.5094 & 0.6279 & 0.5835 \\
\hline $\mathrm{UKF}_{\bar{p} 1}(\lambda=0.5)$ & 0.7576 & 0.9355 & 0.8535 & 0.4839 & 0.7647 & 0.5962 & 0.5094 & 0.6279 & 0.5836 \\
\hline $\mathrm{UKF}_{\bar{p} 1}(\lambda=1)$ & 0.7576 & 0.9333 & 0.8572 & 0.4839 & 0.7059 & 0.6001 & 0.5200 & 0.6279 & 0.5830 \\
\hline $\mathrm{UKF}_{\bar{p} 1}(\lambda=5)$ & 0.6970 & 0.8125 & 0.7546 & 0.4194 & 0.5938 & 0.5000 & 0.5682 & 0.6486 & 0.6062 \\
\hline $\mathrm{UKF}_{\bar{p} 1}(\lambda=10)$ & 0.5758 & 0.7576 & 0.6810 & 0.3226 & 0.5000 & 0.4066 & 0.5676 & 0.7059 & 0.6369 \\
\hline
\end{tabular}

filtering performance than the UKF in some runs, they are comparable in terms of TPR, FPR, and PPV.

Based on the above tests, in the rest of the paper, only the UKF is used.

\subsubsection{Comparison of the UKF and the UKF incorporating the prior information}

As mentioned above, the UKF is used as a typical filter to compare the performance with and without the prior information.

Incorporating existing network information The following prior existing network information is assumed to be known: 1) gene1, gene5, and gene7 have little possibility to regulate gene2; 2) gene2, gene3, gene8 have little possibility to regulate gene7. Hence, the indicator matrix in (50) is given by

$$
\boldsymbol{E}=\left(\begin{array}{llllllll}
0 & 0 & 0 & 0 & 0 & 0 & 0 & 0 \\
1 & 0 & 0 & 0 & 1 & 0 & 1 & 0 \\
0 & 0 & 0 & 0 & 0 & 0 & 0 & 0 \\
0 & 0 & 0 & 0 & 0 & 0 & 0 & 0 \\
0 & 0 & 0 & 0 & 0 & 0 & 0 & 0 \\
0 & 0 & 0 & 0 & 0 & 0 & 0 & 0 \\
0 & 1 & 1 & 0 & 0 & 0 & 0 & 1 \\
0 & 0 & 0 & 0 & 0 & 0 & 0 & 0
\end{array}\right) .
$$

The comparison of the UKF and the UKF incorporating the existing network information (denoted by $\mathrm{UKF}_{p 1}$ ) with $\lambda=2$ is shown in Table 3. It can be seen that the

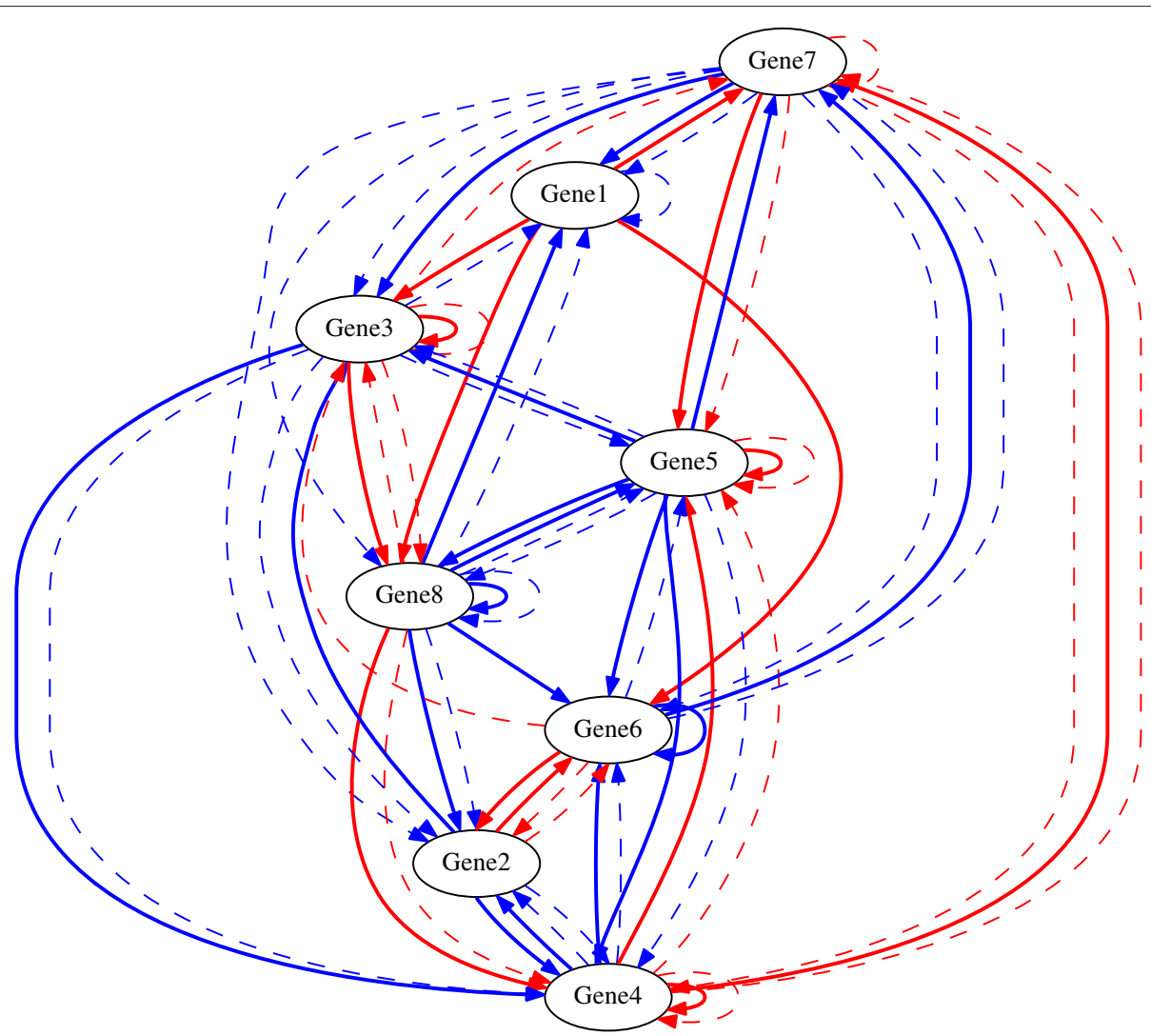

Figure 1 Inferred regulations of $\mathrm{UKF}_{p 2}$ and true regulations. 


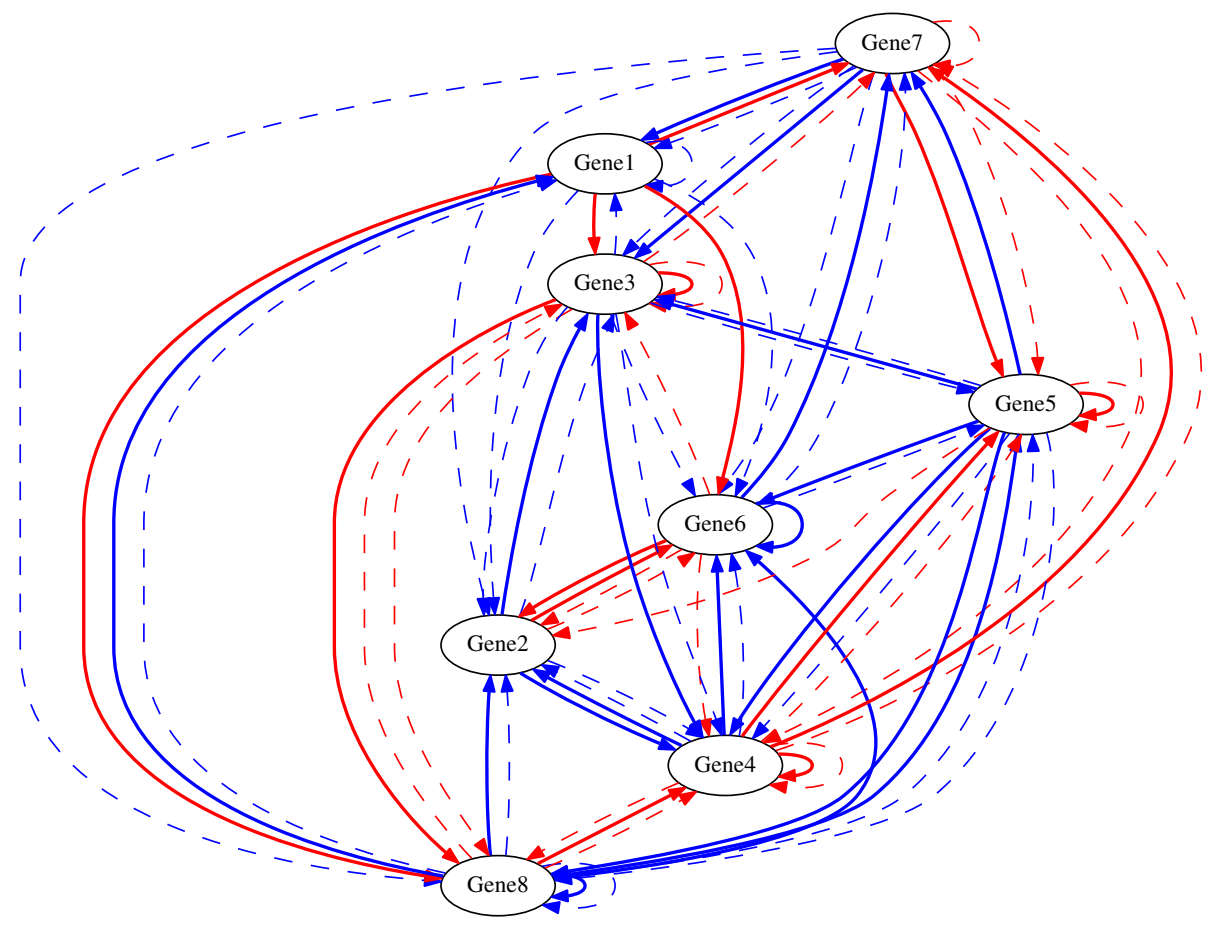

Figure 2 Inferred regulations of UKF and true regulations.

average $\mathrm{TP} \#$ and $\mathrm{TN} \#$ of the $\mathrm{UKF}_{p 1}$ are both higher than those of the UKF. In addition, the average FP\# and FN\# of the $\mathrm{UKF}_{p 1}$ are lower than those of the UKF. Hence, the $\mathrm{UKF}_{p 1}$ predicts more correct links and nonlinks than the UKF. Moreover, the $\mathrm{UKF}_{p 1}$ produces less incorrect links and missed links than the UKF. The average TPR and the precision of the $\mathrm{UKF}_{p 1}$ are higher than those of the UKF. In addition, the average FPR of the $\mathrm{UKF}_{p 1}$ is lower than that of the UKF. Hence, by using the existing network information, the inference accuracy can be improved.

The performance of $\mathrm{UKF}_{p 1}$ with different $\lambda$ is shown in Table 4. It is seen that the performance of $\mathrm{UKF}_{p 1}$ and UKF is close when $\lambda$ is small since only small regulation is imposed on the solution. When $\lambda$ is large, the difference between the $\mathrm{UKF}_{p 1}$ and UKF is large. In particular, the $\mathrm{UKF}_{p 1}$ provides sparser solution than the UKF when $\lambda$ is large. It can be seen from Table 4 , the average FPR of $\mathrm{UKF}_{p 1}$ decreases with the increasing of $\lambda$. The average TPR of $\mathrm{UKF}_{p 1}$, however, does not increase monotonically with the increasing of $\lambda$. The average PPR of $\mathrm{UKF}_{p 1}$ increases with the increasing of $\lambda$. Hence, roughly speaking, the $\mathrm{UKF}_{p 1}$ is better than the UKF when large $\lambda$ is used.

To consider the strength of the links, rather than setting it to $1, e_{i j}$ (in the indicator matrix $\boldsymbol{E}$ ) is set to different values. Large $e_{i j}$ is used if the strength of the link from gene $j$ to gene $i$ is strong. For convenience, the UKF considering the strength of links is denoted as $\mathrm{UKF}_{\hat{p} 1}$. To compare the performance of $\mathrm{UKF}_{\hat{p} 1}$ with $\mathrm{UKF}_{p 1}$, for $\mathrm{UKF}_{\hat{p} 1}$, the values of the second row in Equation (79) is multiplied by 5 . The performance of $\mathrm{UKF}_{\hat{p} 1}$ using different $\lambda$ is given in Table 5 . It can be seen from Tables 4 and 5 that the performance of $\mathrm{UKF}_{\hat{p} 1}$ and $\mathrm{UKF}_{p 1}$ is close when $\lambda$ is small, e.g., $\lambda=0.1$. In addition, the average TPR and FPR of $\mathrm{UKF}_{\hat{p} 1}$ is smaller

Table 7 Comparison of $\mathrm{UKF}_{p 2}$ using different $\lambda$

\begin{tabular}{lcccccccrr}
\hline & \multicolumn{3}{c}{ True positive rate } & \multicolumn{3}{c}{ False positive rate } & \multicolumn{2}{c}{ Positive predictive rate } \\
\hline Filters & Min & Max & Avg & Min & Max & Avg & Min & Max & Avg \\
\hline $\operatorname{UKF}_{p 2}(\lambda=0.1)$ & 0.7576 & 0.9355 & 0.8304 & 0.4839 & 0.6970 & 0.5699 & 0.5306 & 0.6512 & 0.5914 \\
\hline $\operatorname{UKF}_{p 2}(\lambda=0.5)$ & 0.6970 & 0.8710 & 0.7750 & 0.4194 & 0.5758 & 0.4902 & 0.5682 & 0.6585 & 0.6198 \\
\hline $\operatorname{UKF}_{p 2}(\lambda=1)$ & 0.6970 & 0.7879 & 0.7335 & 0.4194 & 0.5000 & 0.4462 & 0.5897 & 0.6667 & 0.6355 \\
\hline $\operatorname{UKF}_{p 2}(\lambda=5)$ & 0.4545 & 0.6667 & 0.5501 & 0.3226 & 0.4516 & 0.3791 & 0.5714 & 0.6471 & 0.6064 \\
\hline $\operatorname{UKF}_{p 2}(\lambda=10)$ & 0.4545 & 0.5455 & 0.4800 & 0.2903 & 0.3871 & 0.3523 & 0.5556 & 0.6538 & 0.5920 \\
\hline
\end{tabular}




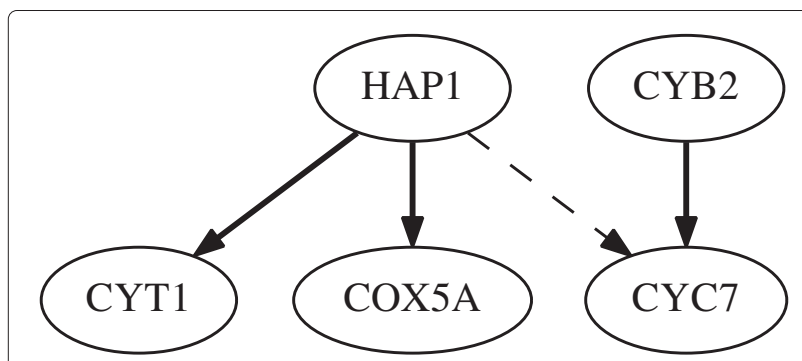

Figure 3 Pathway model of the five genes in yeast protein synthesis network.

than that of $\mathrm{UKF}_{p 1}$ for all tested $\lambda$ except for $\lambda=0.1$. Hence, PPR is used to evaluate the performance of $\mathrm{UKF}_{\hat{p} 1}$ and $\mathrm{UKF}_{p 1}$. Although the average PPR of $\mathrm{UKF}_{\hat{p} 1}$ and $\mathrm{UKF}_{p 1}$ is close when the $\lambda$ is large, e.g., $\lambda=10$, the average $\mathrm{PPR}$ of $\mathrm{UKF}_{\hat{p} 1}$ is consistently higher than that of $\mathrm{UKF}_{p 1}$. The results indicate that the inference accuracy of $\mathrm{UKF}_{\hat{p} 1}$ and $\mathrm{UKF}_{p 1}$ are close when $\lambda$ is very small or very large. The inference accuracy of $\mathrm{UKF}_{\hat{p} 1}$ outperforms $\mathrm{UKF}_{p 1}$ when the appropriate strength of the link and parameter $\lambda$ are used.
To consider the effect of false prior knowledge, the second row of the indicator matrix in Equation (79) is changed to $[0,1,1,1,0,1,0,1]$, which conflicts with the truth. For convenience, we use $\mathrm{UKF}_{\bar{p} 1}$ to denote the UKF incorporating this false prior knowledge. In Table 6, the performance of $\mathrm{UKF}_{\bar{p} 1}$ with different $\lambda$ is shown. It can be seen from Tables 4 and 6 that the average TPR of $\mathrm{UKF}_{\bar{p} 1}$ is smaller than that of $\mathrm{UKF}_{p 1}$ when $\lambda$ is small, e.g., $\lambda=$ $0.1,0.5$. In addition, the average FPR of $\operatorname{UKF}_{\bar{p} 1}$ is larger than that of $\mathrm{UKF}_{p 1}$ when $\lambda$ is large, e.g., $\lambda=5,10$. Moreover, although the average PPR of $\mathrm{UKF}_{\bar{p} 1}$ is close to that of $\mathrm{UKF}_{p 1}$ when $\lambda$ is small, the average PPR of $\mathrm{UKF}_{\bar{p}}$ is consistently lower than that of $\mathrm{UKF}_{p 1}$. Hence, as expected, the results indicate that the false prior knowledge will lead to worse inference result.

Incorporating LASSO The problem setup is the same as before except that the LASSO rather than the existing network information is used. The UKF incorporating LASSO is denoted as $\mathrm{UKF}_{p 2}$.

As shown in Table 3, the average TP\# and FP\# of $\mathrm{UKF}_{p 2}$ are lower than those of UKF and the average TN\# and FN\# of $\mathrm{UKF}_{p 2}$ are higher than those of UKF. Hence, $\mathrm{UKF}_{p 2}$ produces less links, including correct and incorrect ones.
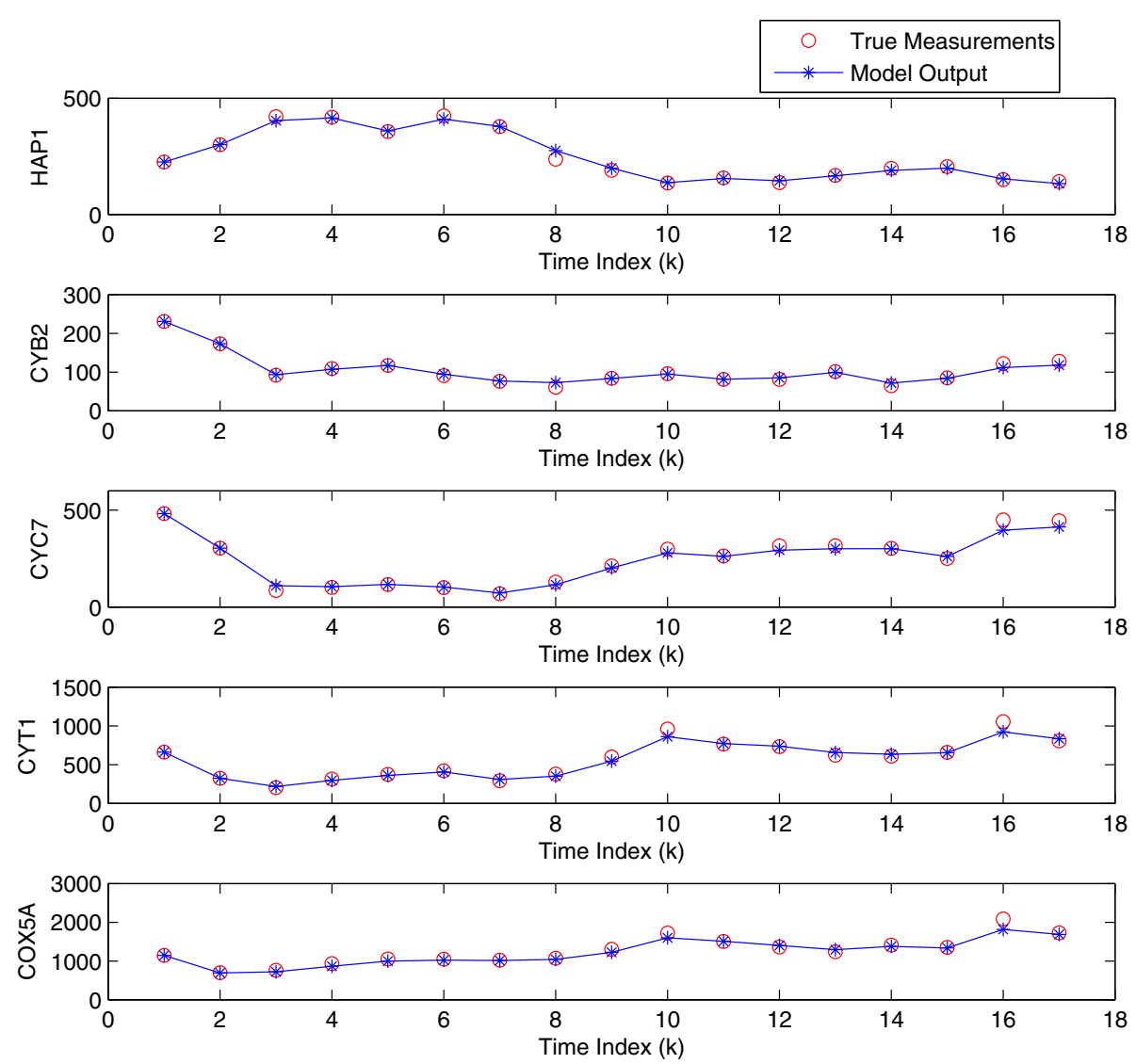

Figure 4 True gene expression and model output. 

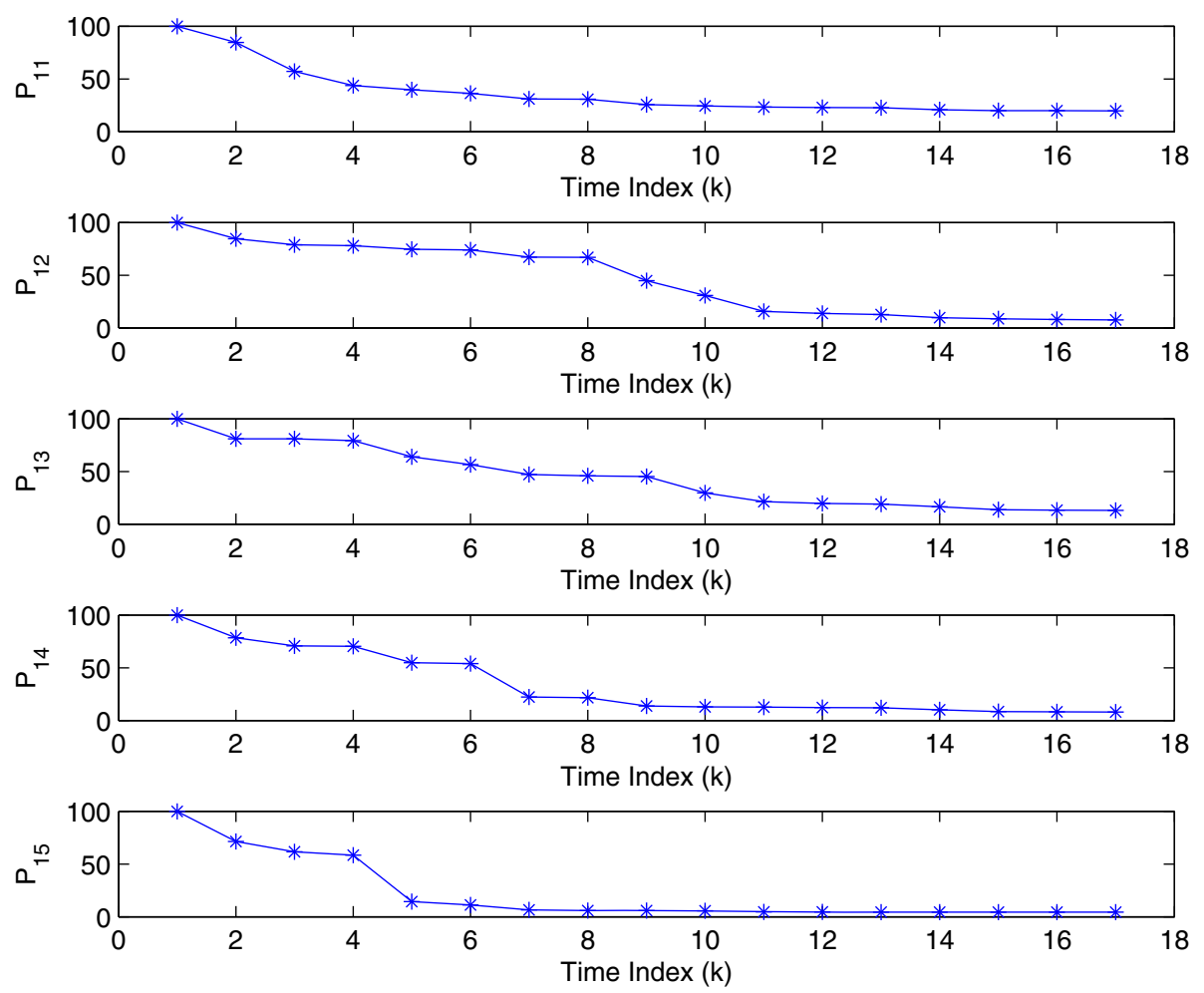

Figure 5 Variance of regulatory coefficients.

In addition, $\mathrm{UKF}_{p 2}$ produces more nonlinks and missed links. It is consistent with the fact that the LASSO tends to provide a sparse solution. It can be seen from Table 3 that the average FPR of $\mathrm{UKF}_{p 2}$ is lower than that of UKF and the average precision of $\mathrm{UKF}_{p 2}$ is higher than that of UKF. Hence, by incorporating LASSO, the inference accuracy is improved.

A representative inference result of $\mathrm{UKF}_{p 2}$ and the true regulations are shown in Figure 1. For comparison, the inference result of UKF and the true regulations are shown in Figure 2. By comparing Figure 2 and Figure 1, it can be seen that UKF falsely predicts the nonlinks from gene1 to gene2, from gene3 to gene6, from gene4 to gene8, from gene5 to gene2, and from gene6 to gene4 while $\mathrm{UKF}_{p 2}$ does not.

The performance $\mathrm{UKF}_{p 2}$ with different $\lambda$ is shown in Table 7. It is seen that the performance of $\mathrm{UKF}_{p 2}$ and
UKF is close when $\lambda$ is small since only small regulation is imposed on the solution. When $\lambda$ is large, the difference between $\mathrm{UKF}_{p 2}$ and UKF is large. The average TPR and FPR of UKF ${ }_{p 2}$ decrease with the increasing of the $\lambda$. The average PPR does not increase monotonicallly with the increasing of $\lambda$. Generally speaking, for different $\lambda, \mathrm{UKF}_{p 2}$ is more sensitive than that of $\mathrm{UKF}_{p 1}$. Although the performance of $\mathrm{UKF}_{p 2}$ depends on $\lambda$, the average PPR of $\mathrm{UKF}_{p 2}$ is consistently higher than that of UKF. Hence, roughly speaking, $\mathrm{UKF}_{p 2}$ has better performance than UKF.

Incorporating the range constraint The existing network information can be used to provide the rough range constraint of $\bar{x}$. A tight constraint is forced on the regulation coefficient $a_{i j}$ when there is a small regulation possibility from genej to gene $i$ and a loose constraint is forced on the regulation coefficient with no prior information.

Table 8 Inferred results of the UKF and UKF $_{p 2}$

\begin{tabular}{|c|c|c|c|c|}
\hline Filters & True positives \# & False positives \# & True negatives \# & False negatives \# \\
\hline UKF & 1 & 7 & 14 & 3 \\
\hline $\mathrm{UKF}_{p 2}$ & 2 & 3 & 18 & 2 \\
\hline Filters & \multicolumn{2}{|c|}{ TPR } & FPR & Precision \\
\hline UKF & \multicolumn{2}{|c|}{0.25} & 0.3333 & 0.1250 \\
\hline $\mathrm{UKF}_{p 2}$ & \multicolumn{2}{|c|}{0.5000} & 0.1429 & 0.4000 \\
\hline
\end{tabular}


In the simulation, for the coefficients corresponding to the zero elements in (79), the lower bound and the upper bound are set as -10 and 10 , respectively. For the coefficients corresponding to the nonzero elements in (79), the lower bound and the upper bound are set as -0.1 and 0.1 , respectively. The UKF incorporating the range constraint is denoted as $\mathrm{UKF}_{p 3}$. As shown in Table 3, the average FPR of $\mathrm{UKF}_{p 3}$ is lower than that of UKF and the average precision of $\mathrm{UKF}_{p 3}$ is higher than that of UKF.

\subsection{Yeast protein synthesis network}

In this section, time-series gene expression data of the yeast protein synthesis network is used. Five genes (HAP1, CYB2, CYC7,CYT1, and COX5A) of the yeast protein synthesis network are considered and 17 data points which can be found in [35] are collected. The regulation relationship between them has been revealed by the biological experiment and shown in Figure 3. The dashed arrow in Figure 3 denotes 'repression' and the solid arrow denotes 'activation.'

The GRN is inferred by the UKF and $\mathrm{UKF}_{p 2}$. The predicted gene expressions using parameters estimated by $\mathrm{UKF}_{p 2}$ and the true measured gene expressions are shown in Figure 4. It can be seen that the model output fits the measured data well. The variances of the regulatory coefficients of HAP1 $\left(P_{1 i}(1 \leq i \leq 5)\right)$ are shown in Figure 5 . It can be seen that the filter converges since the variance $P_{1 i}$ approaches zero. The results for other regulatory coefficients are similar and not shown here. The evaluation of the inferred GRN by UKF and $\mathrm{UKF}_{p 2}$ is shown in Table 8.

By incorporating the sparsity constraint, $\mathrm{UKF}_{p 2}$ provides much better inference results than UKF. As shown in Table 8, the TP\# and TN\# of UKF $p 2$ are higher than those of UKF and the FP\# and FN\# are lower than those of UKF. In addition, it can be seen from Table 8, the FPR of $\mathrm{UKF}_{p 2}$ is lower than that of UKF and the TPR and the precision of $\mathrm{UKF}_{p 2}$ is higher than that of UKF.

\section{Conclusions}

In this paper, we have proposed a framework of employing the point-based Gaussian approximation filters which incorporates the prior knowledge to infer the gene regulatory network (GRN) based on the gene expression data. The performance of the proposed framework is tested by a synthetic network and the yeast protein synthesis network. Numerical results show that the inference accuracy of the GRN by the proposed point-based Gaussian approximation filter incorporating the prior information is higher than using the traditional filters without incorporating prior knowledge. The proposed method works for small- and medium-size GRNs due to the computational complexity considerations. It remains a future research topic how to adapt the proposed inference framework to handle large GRNs at reasonable computational complexity.

\section{Competing interests}

All authors declare that they have no competing interests.

\section{Author details}

${ }^{1}$ Intelligent Fusion Technology, Germantown, MD 20876, USA. ²Department of Electrical Engineering, Columbia University, New York, NY 10027, USA.

Received: 25 July 2013 Accepted: 11 November 2013

Published: 17 December 2013

\section{References}

1. M Zou, SD Conzen, A new dynamic Bayesian network (dbn) approach for identifying gene regulatory networks from time course microarray data. Bioinformatics 21(1), 71-79 (2005)

2. X Zhou, X Wang, R Pal, I Ivanov, M Bittner, ER Dougherty, A Bayesian connectivity-based approach to constructing probabilistic gene regulatory networks. Bioinformatics 20(17), 2918-2927 (2004)

3. M Quach, N Brunel, F d'Alché Buc, Estimating parameters and hidden variables in non-linear state-space models based on odes for biological networks inference. Bioinformatics 23(23), 3209-3216 (2007)

4. Z Wang, X Liu, Y Liu, J Liang, V Vinciotti, An extended Kalman filtering approach to modeling nonlinear dynamic gene regulatory networks via short gene expression time series. Comput. Biol. Bioinformatics, IEEE/ACM Trans. 6(3), 410-419 (2009)

5. X Wu, P Li, N Wang, P Gong, EJ Perkins, Y Deng, C Zhang, State space model with hidden variables for reconstruction of gene regulatory networks. BMC Syst Biol. 5(Suppl 3), S3 (2011)

6. AV Werhli, D Husmeier, Reconstructing gene regulatory networks with Bayesian networks by combining expression data with multiple sources of prior knowledge. Stat. Appl. Genet. Mol. Biol. 6, Article 15 (2007)

7. J Mazur, D Ritter, G Reinelt, L Kaderali, Reconstructing nonlinear dynamic models of gene regulation using stochastic sampling. BMC Bioinformatics 10, 448 (2009)

8. A Noor, E Serpedin, M Nounou, $\mathrm{H}$ Nounou, Inferring gene regulatory networks via nonlinear state-space models and exploiting sparsity. Comput. Biol. Bioinformatics, IEEE/ACM Trans. 9(4), 1203-1211 (2012)

9. M Hecker, S Lambeck, S Toepfer, E van Someren, R Guthke, Gene regulatory network inference: Data integration in dynamic models - a review. Biosystems 96(1), 86-103 (2009)

10. F Markowetz, R Spang, Inferring cellular networks - a review. BMC Bioinformatics 8(Suppl 6), S5 (2007)

11. Y Huang, I Tienda-Luna, Y Wang, Reverse engineering gene regulatory networks. Signal Process. Mag., IEEE 26(1), 76-97 (2009)

12. $\mathrm{H}$ de Jong, Modeling and simulation of genetic regulatory systems: a literature review. J. Comput. Biol. 9, 67-103 (2002)

13. SJ Julier, JK Uhlmann, Unscented filtering and nonlinear estimation. Proc. IEEE 92(3), 401-422 (2004)

14. K Ito, KXiong, Gaussian filters for nonlinear filtering problems. Automatic Control, IEEE Trans. 45(5), 910-927 (2000)

15. I Arasaratnam, S Haykin, Cubature kalman filters. Automatic Control, IEEE Trans. 54(6), 1254-1269 (2009)

16. B Jia, M Xin, Y Cheng, Sparse-grid quadrature nonlinear filtering Automatica 48(2), 327-341 (2012)

17. M Arulampalam, S Maskell, N Gordon, T Clapp, A tutorial on particle filters for online nonlinear/non-Gaussian Bayesian tracking. Signal Process., IEEE Trans. 50(2), 174-188 (2002)

18. X Shen, $\mathrm{H}$ Vikalo, Inferring parameters of gene regulatory networks via particle filtering. EURASIP J. Adv. Signal Process. 2010, 204612 (2010)

19. E Steele, A Tucker, PA 't Hoen, M Schuemie, Literature-based priors for gene regulatory networks. Bioinformatics 25(14), 1768-1774 (2009)

20. S Christley, Q Nie, XXie, Incorporating existing network information into gene network inference. PLoS ONE 4(8), e6799 (2009)

21. Y Tamada, S Kim, H Bannai, S Imoto, K Tashiro, S Kuhara, S Miyano, Estimating gene networks from gene expression data by combining 
Bayesian network model with promoter element detection. Bioinformatics 19(suppl 2), 227-236 (2003)

22. H Li, M Zhan, Unraveling transcriptional regulatory programs by integrative analysis of microarray and transcription factor binding data. Bioinformatics 24(17), 1874-1880 (2008)

23. N Bouaynaya, R Shterenberg, D Schonfeld, Methods for optimal intervention in gene regulatory networks [applications corner]. Signal Process. Mag., IEEE 29(1), 158-163 (2012)

24. L Chen, K Aihara, Chaos and asymptotical stability in discrete-time neural networks. Physica D: Nonlinear Phenomena 104(3), 286-325 (1997)

25. L Qian, H Wang, ER Dougherty, Inference of noisy nonlinear differential equation models for gene regulatory networks using genetic programming and Kalman filtering. Signal Process., IEEE Trans. 56(7), 3327-3339 (2008)

26. J Vohradsky, Neural model of the genetic network. J. Biol. Chem. 276(39), 36168-36173 (2001)

27. E Mjolsness, T Mann, R Castano, B Wold, From coexpression to coregulation: an approach to inferring transcriptional regulation among gene classes from large-scale expression data. in Advances in Neural Information Processing Systems 12, 928-934 (1999)

28. M Nørgaard, NK Poulsen, O Ravn, New developments in state estimation for nonlinear systems. Automatica 36(11), 1627-1638 (2000)

29. IP Mysovskikh, The Approximation of Multiple Integrals by Using Interpolatory Cubature Formulae in Quantitative Approximation, ed. by R DeVore, K Scherer (Academic Press, New York, 1980)

30. AH Jazwinski, Stochastic Processes and Filtering Theory (Academic Press Inc., Waltham, MA, 2007)

31. BO Teixeira, LA Tôrres, LA Aguirre, DS Bernstein, On unscented Kalman filtering with state interval constraints. J. Process Control 20(1), 45-57 (2010)

32. S Wright, R Nowak, M Figueiredo, Sparse reconstruction by separable approximation. Signal Process., IEEE Trans. 57(7), 2479-2493 (2009)

33. D Simon, DL Simon, Constrained Kalman filtering via density function truncation for turbofan engine health estimation. Int. J. Syst. Sci. $\mathbf{4 1}$ (2), 159-171 (2010)

34. F Emmert-Strib, M Dehmer, Analysis of Microarray Data (Wiley-Blackwell, Hoboken, NJ, 2008)

35. H Wang, L Qian, E Dougherty, Inference of gene regulatory networks using s-system: a unified approach. Syst. Biol., IET 4(2), 145-156 (2010)

doi:10.1186/1687-4153-2013-16

Cite this article as: Jia and Wang: Gene regulatory network inference by point-based Gaussian approximation filters incorporating the prior information. EURASIP Journal on Bioinformatics and Systems Biology 2013 2013:16.

\section{Submit your manuscript to a SpringerOpen ${ }^{\mathcal{O}}$ journal and benefit from:}

- Convenient online submission

- Rigorous peer review

- Immediate publication on acceptance

- Open access: articles freely available online

- High visibility within the field

- Retaining the copyright to your article

Submit your next manuscript at $\boldsymbol{\wedge}$ springeropen.com 